This item was submitted to Loughborough's Research Repository by the author.

Items in Figshare are protected by copyright, with all rights reserved, unless otherwise indicated.

\title{
A study on the influences of tyre lags and suspension damping on the instantaneous response of a vehicle
}

PLEASE CITE THE PUBLISHED VERSION

http://dx.doi.org/10.1243/09544070JAUTO671

PUBLISHER

SAGE @ IMechE

VERSION

AM (Accepted Manuscript)

LICENCE

CC BY-NC-ND 4.0

REPOSITORY RECORD

Mavros, George. 2014. "A Study on the Influences of Tyre Lags and Suspension Damping on the Instantaneous Response of a Vehicle”. figshare. https://hdl.handle.net/2134/14753. 
This item was submitted to Loughborough's Institutional Repository (https://dspace.lboro.ac.uk/) by the author and is made available under the following Creative Commons Licence conditions.

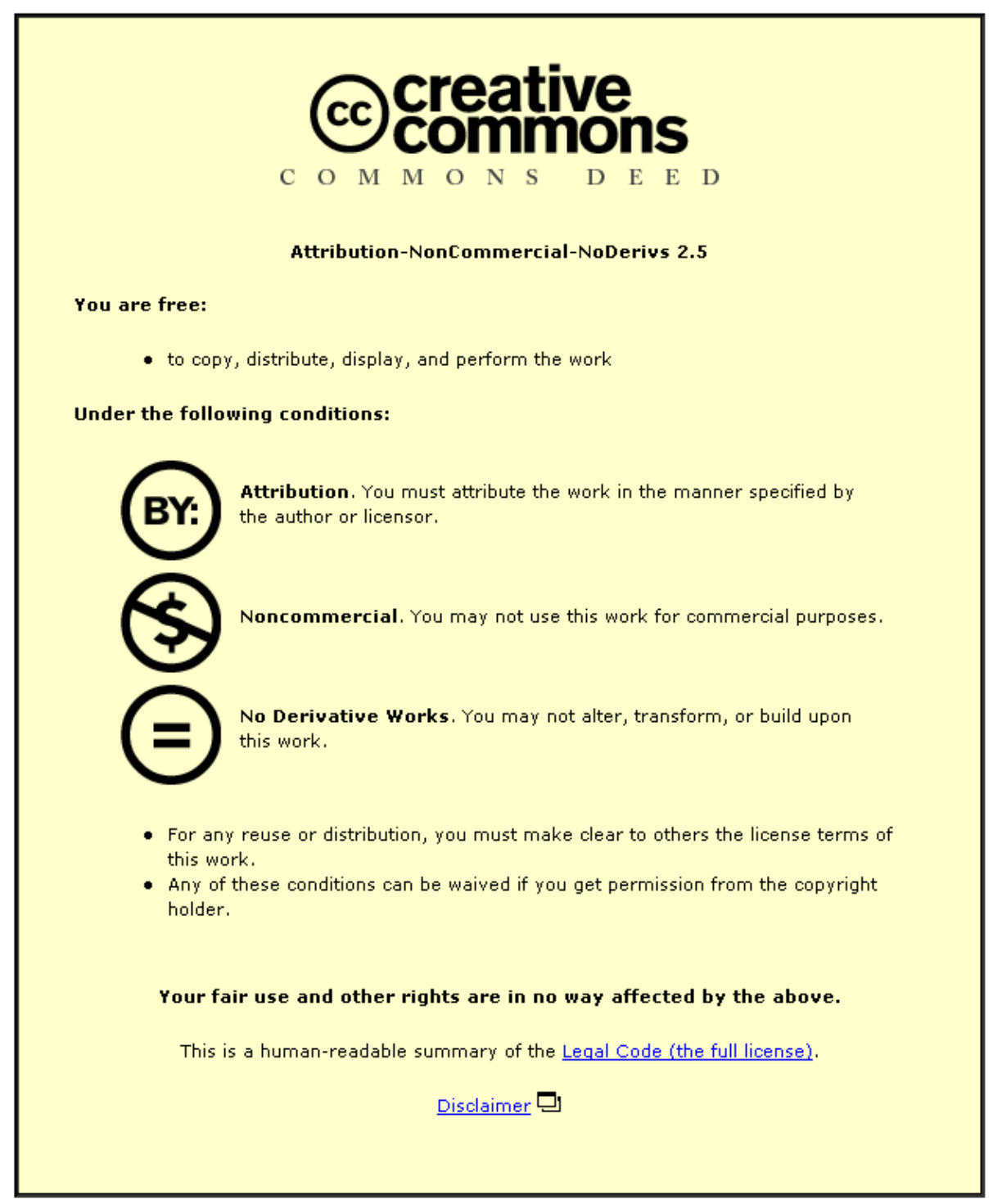

For the full text of this licence, please go to: http://creativecommons.org/licenses/by-nc-nd/2.5/ 
A study on the influence of tyre lags and suspension damping on the instantaneous response of a vehicle

\author{
G. MAVROS*†
}

†School of Aeronautical and Automotive Engineering, Loughborough University

Contact Details:

Dr George Mavros

Aeronautical and Automotive Engineering

Loughborough University

Loughborough

Leicestershire

LE11 3TU

UK

Direct line: +44(0)1509 227273

Fax: +44(0)1509 227275

e-mail: g.mavros@lboro.ac.uk 


\begin{abstract}
The paper aims to quantify the influence of non-steady-state tyre behaviour and suspension damping on the instantaneous response of a vehicle to handling manoeuvres. The influence of such parameters cannot be determined using steady-state tests. In the present work, a recently developed methodology is employed, which has been applied in the past to simple transient handling studies. Here, the approach is applied to more demanding situations and the effects of tyre lags and suspension damping are directly related to the under-, over, or neutral-steer character of the vehicle under test. To distinguish the effect of tyre force lags, tyre non-linearities and combined tyre-vehicle non-linearities, an increasingly complex approach is adopted, which involves the implementation of a linear bicycle-model, a nonlinear bicycle-model and a 10-DOF non-linear vehicle model. In all cases tyre force lags are simulated using the relaxation length concept. The 10-DOF vehicle model is validated against experimental measurements and forms the basis for fully non-linear studies. It is shown that even inherently under-steering vehicles may initially over-steer as a result of the delayed response of tyres. Finally, it is demonstrated that the proposed approach captures the effect of suspension damping and some problems are discussed regarding its application.
\end{abstract}

Keywords: Transient handling; Tyre force lag; Suspension damping; Non-linear model; 


\section{Introduction}

The stability of the perturbed motion of linear and non-linear vehicles has been studied in the past and is well documented [1-5]. These studies aim primarily to determine the mathematical conditions that induce instability in a vehicle's motion. Hence, the characterisation of the transient handling response is usually limited to determining whether a vehicle is stable or unstable. In many cases, transient responses are empirically assessed and compared with each-other in the time domain, using parameters such as the rise-time of the yaw-rate response to a step-steer input [5]. In any case, the characterisation of a vehicle as under-, over-, or neutral-steering has only been defined for steady-state operating conditions. Recently, a framework has been developed [6], [7] which extends the applicability of the aforementioned characterisations/terms in transient operating conditions. The methodology is based on the definition of an assumedly 'neutral' behaviour under transient conditions and the subsequent comparison of the instantaneous behaviour of a vehicle with this 'neutral' behaviour. The comparison is performed using a newly defined measure, termed the 'normalised yaw impulse’ [6].

It is a natural consequence to attempt to employ such a methodology in order to assess the influence of important parameters such as the transient behaviour of tyres or suspension damping, on the instantaneous response of a vehicle to abrupt manoeuvres. Pacejka [5] provides a detailed study of the handling behaviour of a linear bicycle model with transient tyres. In this study, non-steady-state tyre behaviour is accounted for by the relaxation length concept [5]. A frequency domain analysis shows a minor influence of the tyre force 
first order lag on the response of the vehicle to steering inputs, which, as expected, vanishes with increasing forward speed.

The work presented in this paper examines the transient handling response in the time domain. Within the short time-span of a transient handling response, the driver develops a 'feeling' of the handling qualities of a vehicle. During this period, the influence of transient tyre behaviour and damping should be evident. In what follows, this influence is quantified and some interesting observations are made.

\section{The transient handling assessment approach}

The transient handling assessment methodology is presented in detail in references [6] and [7]. The fundamental concept is introduced in [6] where a linear bicycle model is subjected to step-steer excitations. In [6] the extension of the use of steady-state terminology (under-, over- and neutral-steer) into transient operating conditions is attempted. In [7] the method is applied to a bicycle model with non-linear tyre forces and it is demonstrated that, if the input is severe enough, the vehicle's character might change during the course of a transient manoeuvre, following precisely the changes predicted by the steady-state handling diagram [2],[5]. A brief overview of the handling assessment method is provided in the present section in order to facilitate understanding of the results presented in subsequent sections.

The assessment of the instantaneous handling behaviour of a vehicle subjected to a transient steering excitation is achieved using two related measures, namely the dynamic and neutral kinematic normalised yaw impulses, denoted $\Gamma_{d}$ and $\Gamma_{k n}$, respectively. The dynamic normalised impulse is defined as: 


$$
\Gamma_{d}=\frac{|\mathrm{a}| \sum_{i=1,2} F_{y i} \cdot \mathrm{d} t-|\mathrm{b}| \sum_{i=3,4} F_{y i} \cdot \mathrm{d} t}{|\mathrm{a}| \sum_{i=1,2} F_{y i} \cdot \mathrm{d} t+|\mathrm{b}| \sum_{i=3,4} F_{y i} \cdot \mathrm{d} t}
$$

where each lateral force in equation (1) represents the projection of the net tyre force of a possibly steered and braking tyre on the y axis of the SAE vehicle frame of reference [8]. In the case of a linear bicycle model, the above relation can be simplified and expressed as a function of kinematic quantities and a parameter, $\rho$ [6]:

$\Gamma_{d}=\Gamma_{k}=\frac{U \delta-V(1-\rho)-r(|\mathrm{a}|+\rho|\mathrm{b}|)}{U \delta-V(1+\rho)-r(|\mathrm{a}|-\rho|\mathrm{b}|)}$

where $\Gamma_{k}$ denotes the kinematic normalised yaw impulse and parameter $\rho$ is given below:

$$
\rho=\frac{|\mathrm{b}| C_{r}}{|\mathrm{a}| C_{f}}
$$

For a neutral vehicle $\rho=1$, and $\Gamma_{k}$ can be simplified further, resulting in the neutral kinematic normalised yaw impulse:

$$
\Gamma_{k}=\Gamma_{k n}=\frac{U \delta-r l}{U \delta-2 V-r(|a|-|b|)}
$$


The underlying principle for the assessment of transient responses is the axiomatic declaration that a linear neutral bicycle model, without phase lags in the generation of the tyre forces, behaves neutrally under any possible steady-state or transient operating conditions. It is easily seen that, $\Gamma_{k n}$, as calculated for a neutral linear vehicle during the course of a transient manoeuvre, will always be equal to $\Gamma_{d}$. At this point it should be noted that results presented later herein justify the selection of a linear neutral bicycle model without lags in the generation of tyre forces, as the absolute benchmark for assessing transient handling behaviour.

The assessment of the response of a given vehicle is based on the initial assumption that the vehicle is neutral, i.e. it resembles the behaviour of the linear neutral bicycle model. This assumption is checked by calculating $\Gamma_{k n}$ for the assumedly neutral response of the vehicle and subsequently comparing it with the dynamic yaw impulse $\Gamma_{d}$ as calculated by equation (1). If $\Gamma_{k n}$ is found to be equal to $\Gamma_{d}$, then the response is characterised as neutral for as long as the equality holds true [6]. In the event that $\Gamma_{d}$ is smaller than $\Gamma_{k n}$, the response is characterised as under-steering [6], whereas if $\Gamma_{d}$ is greater than $\Gamma_{k n}$, the response is classified as over-steering.

The comparison between $\Gamma_{d}$ and $\Gamma_{k n}$ is performed by employing two different ratios, denoted $Q$ and $Q_{s}$, respectively, each one characterised by its own strengths and weaknesses [6]. The $Q$ ratio is provided by the following relation:

$$
Q=\frac{\Gamma_{d}}{\Gamma_{k n}}
$$


For $Q=1$ the vehicle behaves neutrally. If $Q<1$ it exhibits under-steer, while $Q>1$ points to over-steering. The usefulness of the $Q$ ratio as a handling performance index deteriorates under certain conditions, as a result of $\Gamma_{k n}$ and/or $\Gamma_{d}$ becoming negative [6]. This observation has lead to the definition of the alternative $Q_{s}$ ratio [6], which, although not explicitly used in the present paper is defined below for completeness of the methodology:

$Q_{s}=\frac{\left(\Gamma_{d}-\Gamma_{k n}\right) B_{1}}{\left|\Gamma_{d}-\Gamma_{k n}\right|+B_{2}}$

where $B_{1}$ and $B_{2}$ are Boolean expressions yielding 1 (true) or 0 (false) according to the following relations:

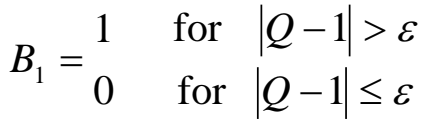

$$
\begin{aligned}
& B_{2}=\begin{array}{lll}
1 & \text { for } & |Q-1| \leq \varepsilon \\
0 & \text { for } & |Q-1|>\varepsilon
\end{array}
\end{aligned}
$$

Parameter $\varepsilon$ represents the 'neutral margin', dictating the maximum relative difference allowed between $\Gamma_{d}$ and $\Gamma_{k n}$, before classifying a transient response as non-neutral.

As long as $|Q-1|=\left|\left(\Gamma_{d}-\Gamma_{k n}\right) / \Gamma_{k n}\right| \leq \varepsilon$, the vehicle is considered neutral, with $B_{1}=0$, $B_{2}=1$ and $Q_{s}=0$. On the other hand, if $|Q-1|=\left|\left(\Gamma_{d}-\Gamma_{k n}\right) / \Gamma_{k n}\right|>\varepsilon$, the relative 
difference between $\Gamma_{d}$ and $\Gamma_{k n}$ is such that the vehicle cannot be characterised as neutral. Under such conditions $B_{1}=1, B_{2}=0$ and $Q_{s}$ represents the sign of the difference between $\Gamma_{d}$ and $\Gamma_{k n}$. If $Q_{s}=-1$ the vehicle exhibits under-steer, whereas if $Q_{s}=1$ it over-steers.

Prior to applying the method for the critical evaluation of the influence of tyre lags and suspension damping, it is worth attempting to further demonstrate the logic behind the approach. As already stated, the approach is based on the comparison of $\Gamma_{d}$ against $\Gamma_{k n}$. For the sake of argument it is assumed that a linear bicycle model without lagging tyre forces is subjected to a transient steering excitation and it is found that $\Gamma_{d}>\Gamma_{k n}$. According to the established criteria, this points towards over-steer. Considering eq. (2) and (4), the relation between $\Gamma_{d}$ and $\Gamma_{k n}$ can be written as follows:

$\frac{U \delta-V(1-\rho)-r(|\mathrm{a}|+\rho|\mathrm{b}|)}{U \delta-V(1+\rho)-r(|\mathrm{a}|-\rho|\mathrm{b}|)}>\frac{U \delta-r l}{U \delta-2 V-r(|a|-|b|)}$

After a series of algebraic manipulations, relation (9) reduces to:

$A \rho>A$

where:

$$
A=V U \delta-U r|\mathrm{~b}| \delta-V^{2}-r V|\mathrm{a}|+r V|\mathrm{~b}|+r^{2}|\mathrm{a}||\mathrm{b}|
$$


Inequality (10) depends on the sign of the term $A$, given by relation (11). While it is difficult to predict the sign of $A$, it can be easily plotted throughout the course of a manoeuvre. Later in section 6 it will be shown that the term $A$ is negative for all bicycle models tested. For the over-steering case this would result in eq. (10) reducing to the final result that $\rho<1$, which immediately points towards over-steer. Hence, it is demonstrated that the result obtained by comparing $\Gamma_{d}$ against $\Gamma_{k n}$ is a sensible way for assessing a vehicle’s transient behaviour, since it points towards the inherent steady-state steering character of the vehicle. However, this argument should by no means be misinterpreted as an assessment of the transient handling behaviour based on the calculation of parameter $\rho$. In fact, parameter $\rho$ can be calculated for any linear bicycle model even without defining the dynamic and kinematic yaw impulses. Dividing the instantaneous lateral forces by the corresponding slip angles suffices in order to find the cornering stiffness and subsequently calculate $\rho$. Even if parameter $\rho$ was indirectly computed at each time-step by comparing equations (1) and (2), for a linear model without tyre lags the resulting value of $\rho$ would be found constant and equal to that calculated apriori using eq. (3). To take the issue further, one could calculate an equivalent parameter $\rho$ for non-linear models or for models with lagging tyre forces. Obviously, the value of $\rho$ would change throughout the course of the manoeuvre. Then, the transient behaviour could be assessed based on the equivalent instantaneous $\rho$, without the need of calculating and comparing the two expressions of the yaw impulse. However, the latter approach is fundamentally different. For example, in reference [6] the $Q$ ratio was employed for the assessment of the response of a linear under-steering vehicle without lagging tyre forces and a constant under-steer coefficient 
(constant $\rho$ ) but varying yaw moment of inertia, $I_{z z}$. It was found that, in transient terms, the amount of under-steer reduced as $I_{z z}$ increased. Clearly, this result could not have been obtained simply by calculating parameter $\rho$. Furthermore, this prediction was confirmed in

[6] by comparing the yaw response of the under-steering vehicles with varying yaw moments of inertia with the response of equivalent neutral models with varying yaw inertia. It was found that the difference between the responses in the time domain reduced as the yaw moment increased, i.e. the under-steer reduced. To conclude, it can be seen that the comparison of $\Gamma_{d}$ against $\Gamma_{k n}$ is a sensible starting point for the assessment of transient handling manoeuvres. Initially, it is found that the characterisation as under-, over-, or neutral-steer by comparing $\Gamma_{d}$ and $\Gamma_{k n}$ is consistent with the inherent steady-state character of a linear vehicle without lagging forces. As will be shown in section 6, the comparison between $\Gamma_{d}$ and $\Gamma_{k n}$ remains valid for models with tyre force lags or equipped with nonlinear tyres. The approach remains generic by adhering to this comparison and avoiding the calculation of parameter $\rho$ which does not capture issues such as the change in transient behaviour due to changes in the yaw moment of inertia [6].

\section{Vehicle modelling}

A number of increasingly complex vehicle models are used in the analysis, namely two versions of a 2-DOF (Degree-Of-Freedom) bicycle model (linear and non-linear), as well as a detailed 10-DOF non-linear vehicle model. In this section only the latter most elaborate model will be presented in detail, as the bicycle model can easily be derived from this one. Hereafter, the 10-DOF model will be referred to as the full-vehicle model. 
The formulation of the equations of motion for the full-vehicle model is based on the Newton-Euler approach, described in [9] and [10]. The sprung vehicle mass possesses six degrees of freedom, consisting of three translations and three rotations in space. The remaining four degrees of freedom represent the rotations of the four wheels about their spin axes. Vertical degrees of freedom for the four un-sprung masses are not considered, as the model is not intended for ride/comfort studies.

The primary six motions of the vehicle body are observed with respect to the moving SAE frame of reference [8], which is attached to the vehicle body. A schematic of the vehicle with the SAE frame of reference and the corresponding six velocities is provided in figure 1. The xz plane of the SAE frame is a plane about which the vehicle is considered geometrically symmetrical. However, the vehicle is not symmetrical about the same plane in terms of its inertial properties. Hence, various products of inertia need not equal zero. Also, the longitudinal position (along the $\mathrm{x}$-axis) of the origin of the SAE frame is assumed to coincide with the longitudinal position of the c.g. (centre of gravity). Considering the above, the equations of motion for the three translations and three rotations of the vehicle body in space, are given below:

$$
\begin{aligned}
& \Sigma F_{x}=m_{T} \cdot(d U / d t-V \cdot r+W \cdot q)-m_{T} \cdot\left[x_{G} \cdot\left(q^{2}+r^{2}\right)-y_{G} \cdot(p \cdot q-d r / d t)-z_{G} \cdot(p \cdot r+d q / d t)\right] \\
& \Sigma F_{y}=m_{T} \cdot(d V / d t-W \cdot p+U \cdot r)-m_{T} \cdot\left[y_{G} \cdot\left(r^{2}+p^{2}\right)-z_{G} \cdot(q \cdot r-d p / d t)-x_{G} \cdot(p \cdot q+d r / d t)\right] \\
& \Sigma F_{z}=m_{S} \cdot(d W / d t-U \cdot q+V \cdot p)-m_{S} \cdot\left[z_{G} \cdot\left(p^{2}+q^{2}\right)-x_{G} \cdot(p \cdot r-d q / d t)-y_{G} \cdot(q \cdot r+d p / d t)\right]
\end{aligned}
$$




$$
\begin{aligned}
& \Sigma M_{x}=I_{x x} \cdot(d p / d t)-\left(I_{y y}-I_{z z}\right) \cdot q \cdot r+I_{y z} \cdot\left(r^{2}-q^{2}\right)-I_{z x} \cdot(p \cdot q+d r / d t) \\
& +I_{x y} \cdot(p \cdot r-d q / d t)+m_{S} \cdot y_{G} \cdot(d W / d t-U \cdot q+V \cdot p)-m_{S} \cdot z_{G} \cdot(d V / d t-W \cdot p+U \cdot r) \\
& \Sigma M_{y}=I_{y y} \cdot(d q / d t)-\left(I_{z z}-I_{x x}\right) \cdot p \cdot r+I_{x z} \cdot\left(p^{2}-r^{2}\right)-I_{x y} \cdot(q \cdot r+d p / d t) \\
& +I_{y z} \cdot(q \cdot p-d r / d t)+m_{S} \cdot z_{G} \cdot(d U / d t-V \cdot r+W \cdot q)-m_{S} \cdot x_{G} \cdot(d W / d t-U \cdot q+V \cdot p) \\
& \Sigma M_{z}=I_{z z} \cdot(d r / d t)-\left(I_{x x}-I_{y y}\right) \cdot p \cdot q+I_{x y} \cdot\left(q^{2}-p^{2}\right)-I_{y z} \cdot(r \cdot p+d q / d t) \\
& +I_{z x} \cdot(r \cdot q-d p / d t)+m_{T} \cdot x_{G} \cdot(d V / d t-W \cdot p+U \cdot r)-m_{T} \cdot y_{G} \cdot(d U / d t-V \cdot r+W \cdot q)
\end{aligned}
$$

Whereas the model does not include provision for vertical motion of the four un-sprung masses at the corners of the vehicle, both the sprung and the total mass, denoted $m_{S}$ and $m_{T}$, respectively, appear in the above equations. In particular, $m_{T}$ appears only in eq. (12), (13), as the longitudinal and lateral DOF are subject to the vehicle’s total inertia. However, when dealing with the vertical motion, it is more appropriate to use the sprung mass, $m_{S}$. Similar comments apply for the use of $m_{S}$ in eq. (15), (16) and $m_{T}$ in eq. (17). The selective implementation of the total, or the sprung mass of the vehicle can be seen as an attempt to improve accuracy in the representation of the dynamics of the vehicle, albeit without introducing additional degrees of freedom, i.e. without increasing the computational effort required. Finally, following the requirement that the longitudinal position of the c.g. lies at the origin of the SAE frame, $x_{G}=0$.

The left-hand-sides of eq. (12)-(17) represent the net forces along the three axes of the SAE frame, or the corresponding moments about the same axes. The primary forces in the $\mathrm{x}$ - and y- directions are generated at the tyre contact patch area. Tyre forces are initially 
considered in the tyre SAE frame [8], however all wheel angles except the steer angle, $\delta$, are assumed small and the projection of tyre local forces to the vehicle SAE frame is calculated by the following relations:

$$
F_{x i}=\left(F_{x t y r e}\right)_{i} \cdot \cos \delta_{i}-\left(F_{y t y r e}\right)_{i} \cdot \sin \delta_{\mathrm{i}}
$$

$$
F_{y i}=\left(F_{x y y r e}\right)_{i} \cdot \sin \delta_{i}+\left(F_{y y y r e}\right)_{i} \cdot \cos \delta_{\mathrm{i}}
$$

The vertical loads at the four corners of the vehicle are calculated as a sum of spring and damper forces, resulting from suspension deflections and their corresponding rates. As an example, the vertical force exerted on the vehicle body by the front-left suspension is calculated as follows:

$$
F_{\text {susp } 1}=-K_{f}\left[\left(z-\left|\frac{t_{r f}}{2}\right| \cdot \theta-|\mathrm{a}| \cdot \phi\right)\right]-D_{f}\left[\left(W-\left|\frac{t_{r f}}{2}\right| \cdot p-|\mathrm{a}| \cdot q\right)\right]
$$

Apart from the forces due to suspension deflection, the rigid reactions to the vehicle body through the suspension links are also considered. The net effect of such reactions is treated using the virtual work method [11]. In particular, it is observed that, as a result of the kinematic properties of the suspension, its primary vertical motion is accompanied by secondary motions in the lateral and forward directions. Thus, an infinitesimal vertical displacement of the tyre contact centre, say $\partial \mathrm{z}_{\text {contact }}$, results in displacements $\partial \mathrm{x}_{\text {contact }}$, $\partial \mathrm{y}_{\text {contact }}$ in the forward and lateral directions, respectively. Now, the suspension may be 
treated as a mechanism with two possible input motion directions, namely longitudinal and lateral. The corresponding output is the associated vertical travel of the tyre contact centre with respect to the vehicle body. If the longitudinal, $F_{x}$, and lateral, $F_{y}$ forces at the centre of the contact patch are known, application of the virtual work method yields the resulting vertical forces applied on the sprung mass, as described by relations (21) and (22).

$$
\begin{aligned}
& F_{z y}=-F_{y} \frac{\partial y_{\text {contact }}}{\partial z_{\text {contact }}} \\
& F_{z x}=-F_{x} \frac{\partial x_{\text {contact }}}{\partial z_{\text {contact }}}
\end{aligned}
$$

Rigid reactions predicted by eq. (21), (22) are added to suspension forces calculated by eq. (20), in order to determine the net vertical forces acting at the four corners of the vehicle. The contribution of tyre shear forces and vertical corner forces is supplemented by that of the vehicle's weight, acting at the c.g. and by aerodynamic drag, acting at the centre of aerodynamic pressure. Depending on the location of their corresponding application point, all aforementioned forces generate moments about the axes of the moving SAE frame of reference. Additional moment about the x-axis is offered by front and rear anti-roll bars. A subtle point should be emphasized here; the roll-resisting moment generated by the anti-roll bars is reacted at the tyre contact patches. Hence, considering again the front left corner, the vertical load carried by the tyre is updated as follows: 


$$
F_{z t y r e 1}=K_{f}\left[\left(z-\left|\frac{t_{r f}}{2}\right| \cdot \theta-|\mathrm{a}| \cdot \phi\right)\right]+D_{f}\left[\left(W-\left|\frac{t_{r f}}{2}\right| \cdot p-|\mathrm{a}| \cdot q\right)\right]-\frac{K_{\text {froll }} \theta}{t_{r f}}
$$

Assuming small vehicle body angles, the orientation of the SAE frame of reference with respect to the road can be considered unchanged and all forces/moments described above can be added to form the left-hand-side terms of eq. (12)-(17). However, this simplification is not adopted here. In particular, the roll and pitch angles are assumed significant and the methodology proposed in [10] for large angles of rotation is employed for their calculation. In turn, these angles form the basis for the construction of an appropriate Euler transformation matrix which is used for the projection of the forces to the instantaneous SAE frame.

Finally, the six primary differential equations of motion (eq. (12)-eq. (17)) are supplemented by four additional equations governing the rotation of the wheels. As an example, the equation of motion of one of the driven wheels is given as:

$$
I_{w} \dot{\omega}=T_{d}-T_{b}-F_{x t y r e} R
$$

Now, the bicycle model is readily available by considering only the lateral and yaw degrees of freedom of the full-vehicle model. Further assuming constant forward speed $U$ and sufficiently small steer angles, eq. (13) and (17) of the full-vehicle model reduce to:

$$
\Sigma F_{y}=Y_{f}\left(a_{f}\right)+Y_{r}\left(a_{r}\right)=m_{T}(d V / d t+U \cdot r)
$$


$\Sigma M_{z}=|a| Y_{f}\left(a_{f}\right)-|b| Y_{r}\left(a_{r}\right)=I_{z z}(d r / d t)$

where $Y_{f}, Y_{r}$ denote the front and rear lateral tyre forces, as functions of the corresponding slip angles.

In the simplified, linear case, $Y_{f}, Y_{r}$ are assumed linear functions of the slip angles:

$Y_{f}\left(a_{f}\right)=C_{f} a_{f}$ and $Y_{r}\left(a_{r}\right)=C_{r} a_{r}$

For the non-linear bicycle model, as well as for the full-vehicle model, tyre forces are calculated using a version of the Magic Formula tyre model [12], as discussed in the following section.

\section{Tyre modelling}

The low bandwidth achieved with steering manoeuvres allows the treatment of transient tyre behaviour in a simplified manner, using a single point contact transient tyre model [5]. Such models consist of a conventional steady-state model, where the steady-state slip angle is replaced by its transient counterpart, which in turn is provided by a first order differential equation based on the relaxation length concept. The steady-state part of the model used in this study is the version of the Magic Formula presented in [12]. The fundamental equations are given below:

$Y(X)=y(x)+S_{V}$ 
with:

$y(x)=D \sin [C \arctan \{B x-E(B x-\arctan B x)\}]$

and

$x=X+S_{H}$

where $X$ represents the primary input variable (in the form of $\tan a$ or $k$ ) and $Y$ represents the primary output variable in the form of lateral/longitudinal force or selfaligning moment.

The primary parameters of the Magic formula, namely $B, C, D, E, S_{H}$ and $S_{V}$, appear as functions of the normal load, $F_{z}$, the camber angle, $\gamma$, and a number of secondary constants [12]. Combined braking/cornering is approached in a simple yet effective manner, using the treatment proposed in [13], which is based on the similarity concept.

Transient tyre behaviour is simulated by substituting the steady state slip values $\tan a, k$ with their transient equivalents, denoted $\tan a^{\prime}, k^{\prime}$. These in turn are calculated by the following differential equations [5]:

$$
\frac{d}{d t} \tan a^{\prime}+\frac{|u|}{\sigma_{y}} \tan a^{\prime}=\frac{v}{\sigma_{y}}
$$




$$
\frac{d}{d t} k^{\prime}+\frac{|u|}{\sigma_{x}} k^{\prime}=\frac{|u|-R \omega \operatorname{sgn}(u)}{\sigma_{x}}
$$

where the absolute value is used so that eq. (31), (32) are valid for rearward rotation of the wheels as well.

Finally, the substitution of steady-state with transient slip angles also applies to eq. (27), yielding the linear tyre forces.

\section{Validation of the full-vehicle model}

The methodology for transient handling assessment discussed in section 2 has been applied for the analysis of trivial manoeuvres performed with simple vehicle models [6], [7]. In an attempt to evaluate the performance of the method under more realistic conditions, the parameters of the full vehicle model were matched those of a Jaguar XJ350 test vehicle. The test vehicle was equipped with an RT3200 GPS/Inertial measurement system which provided the six primary vehicle states. The steer-angle, wheel speed and other useful measures were obtained directly from the vehicle’s CAN network. Since many tests were conducted at constant speed using the cruise control system of the test-vehicle, a simple PID controller was added to the full vehicle model, regulating the torque at the driving wheels. Other additions to the model include the consideration of a static camber angle and toe-in steering adjustments. A significant number of tests were carried out, involving stepsteer and arbitrary-steer inputs at various speeds. Experimentally measured steering inputs 
were then fed into the full-vehicle model and numerical results were compared with the corresponding experimental test-data.

For the purpose of validation of the full vehicle model an arbitrary steering input was selected, as shown in figure 2. The steering input was applied at a forward speed of approximately $13.2 \mathrm{~m} / \mathrm{s}$, which was maintained constant by the cruise control system of the real vehicle, or, alternatively, the PID forward speed controller of the simulation model. Various responses to the given steering input are shown in figs 3-5. Figure 3 depicts the yaw-rate response of both the test-vehicle and the full-vehicle model, figure 4 shows the roll-angle response, while the lateral acceleration response is illustrated in fig. 5. Very good agreement is shown between the model and the actual vehicle. Minor discrepancies can be attributed to the lack of suspension and steering compliance in the model, as well as environmental parameters which might have affected tyre force generation, such as wet patches on the test track. Figure 6 depicts the minor fluctuations observed in the forward speed. It is observed that the PID controller employed for simulation purposes performs better in that it provides a more consistent forward speed. This is due to the fact that in the real vehicle the controller acts through the engine, i.e. engine dynamics are involved in the process of speed control. In contrast, the simple PID controller regulates directly the driving torque applied at the rear wheels. Finally, figure 7 illustrates the vehicle path as obtained from experiment and simulation. Again, very good agreement is observed with the exception of a noticeable difference in the overall direction of motion. This difference is directly attributed to the principle of operation of the GPS/Inertial measurement system. In particular, the path generated by the system includes orientation information, that is, the exact position of the vehicle on the map. Although the initial position of the vehicle has 
been subtracted from the experimental measurements so that the motion starts from point $(0,0)$, this transformation has no effect on the direction of heading. In this case, it appears that at the start of the manoeuvre the actual vehicle was heading towards the north-east compared to the simulation model which, obviously, always heads to the east (positive xaxis) at the start of every manoeuvre. It should be mentioned that the simulation results presented in figures 3-7 are obtained using the nominal vehicle parameters, i.e. without additional tuning to achieve better agreement.

\section{Simulation Results}

The behaviour of the linear and non-linear bicycle models is investigated as a starting point. Three linear vehicles are considered, which are made under-, neutral- and over-steering (based on conventional, steady-state terminology) by adjusting the linear stiffness, $C_{r}$, of the rear tyres. The corresponding parameters of the three bicycle models are shown in Table 1. In all cases a relaxation length $\sigma_{x}=0.3 \mathrm{~m}$ is assumed for both front and rear tyres. To demonstrate the behaviour of the non-linear bicycle model, linear tyre forces are replaced by forces predicted by the Magic Formula, while the relaxation length is kept equal to $0.3 \mathrm{~m}$. All four bicycle-models are subjected to a step-steer input of $0.02 \mathrm{rad}$ at the front wheels. Figure 8 shows the term $A$ (see eqs. (10),(11)) as a function of time for all four models. It is observed that in all cases $A<0$ so that inequality (10) yields instantaneous values for parameter $\rho$ in agreement with the $Q$ ratio response predictions shown in figure 9. It is emphasized again that this should not be misinterpreted as an assessment based on the values of $\rho$. Figure 9 provides the opportunity for additional 
comments. Starting from the under-steering linear model, one can observe a mild oversteering tendency (based on the extension of steady-state terminology to transient conditions, see [6]) at the onset of the manoeuvre. Soon after, the response reduces to under-steer, in agreement with the steady-state expectations. The over-steering tendency relates to the combined effect of front and rear tyre-force lags, which works in favour of the front tyres. This is shown clearly in figure 10 which shows the same under-steering linear bicycle model operating on three different sets of tyres with increasing relaxation length. As the relaxation length increases, the instantaneous behaviour becomes more oversteering, however all three vehicles soon exhibit their steady-state, under-steering character. Returning to figure 9, the neutral bicycle model demonstrates a rather peculiar behaviour, as, in the presence of lagging tyre forces, it is predicted to continuously over-steer. By observing figure 11 which shows the dynamic and kinematic normalised impulses, it becomes apparent that this behaviour is an artefact, attributed to numerical reasons. In particular, both the numerator and denominator of the $Q$ ratio converge to values slightly different from zero and this results in a positive $Q$ ratio. For the neutral bicycle model, after the tyre forces have reached a steady-state, the dynamic yaw impulse will be given by eq. (2), i.e. it is a function of the steady-state tyre forces only. In addition, for the neutral bicycle model, parameter $\rho$ is equal to one, so the dynamic yaw impulse will also be equal to the neutral kinematic yaw impulse given by eq. (4). While theoretically the ratio between dynamic and kinematic yaw impulses should quickly converge to unity, in numerical practice the value of the dynamic yaw impulse is governed by the lagging tyre forces and converges at a slightly slower rate than the kinematic yaw impulse. While this may indicate that the assessment method might be susceptible to such numerical problems, it should be 
emphasized that even vehicles which are marginally non-neutral show a consistent behaviour in terms of the $Q$ ratio, in the presence of any combination of front/rear tyreforce lags. Such issues will be discussed to a further extent later, when studying the response of the full model. The linear responses conclude with that of the over-steering vehicle, which, as expected demonstrates a purely over-steering behaviour. The sudden change of sign observed in figure 5 should not be confused with an indication of shifting towards under-steer. As explained in [6], this is due to the kinematic impulse reducing rapidly and eventually becoming negative. In general, such issues can easily be clarified by observing both the $Q$ ratio and its components (see eq. (5)).

The non-linear bicycle model shows an initial over-steering tendency which then shifts to under-steer. It is noted that the behaviour of the non-linear model, although similar to that of the linear over-steering model, is fundamentally different. In particular, magnification of the area near the peak $Q$ ratio shows that this is a true peak with substantial curvature, not a discontinuity due to a change of sign, as is the case for the over-steering model. Finally, the much more substantial tendency of the non-linear model to over-steer should be attributed to the non-linear tyre model which, based on the position of the c.g. of the bicycle model (see Table 1), predicts a far less under-steering tendency than the linear under-steering model (see also the cornering stiffness included in Table 1).

The full vehicle model is mainly used for the evaluation of the applicability of the proposed methodology under more realistic operating conditions and for the study of the influence of suspension damping. As a case study, the response of the full-vehicle model to an experimentally measured 'imperfect' step-steer input at approximately $13 \mathrm{~m} / \mathrm{s}$ forward speed is investigated. The steering input resulted in a peak lateral acceleration of $7.6 \mathrm{~ms}^{-2}$. 
The steering input is shown in figure 12. To examine the effect of suspension damping, three discrete vehicle set-ups are employed. The nominal set-up uses the parameters of the test-vehicle un-altered. The second set-up involves increasing the damping coefficient at the rear suspension by $50 \%$, while the third one increases the damping at the rear by $100 \%$. The $Q$ ratio response of the nominal and the two hypothetical vehicles is depicted in figure 13, which provides invaluable insight into the applicability of the method in realistic situations. It was shown earlier that eq. (5) might lead to unrealistic conclusions caused by numerical residuals. In such cases, a closer look to the components of eq. (5) immediately reveals the fault. Also, this problem would be caused only by a neutral linear bicycle model with lagging tyre forces, which should be considered impossible to find in practice. Now, figure 13 shows a different type of unwanted behaviour. In particular, prior to the initiation of the steering manoeuvre at approximately 14.2 seconds, significant activity is observed in terms of the $Q$ ratio. This is attributed to minute steering disturbances which are evident prior to the initiation of steering, although not visible in figure 12. Since $Q$ is represented by a ratio, it is expected that it will demonstrate significant sensitivity to the existence of small disturbances either at the numerator, or the denominator. Fortunately, any concerns related to such phenomena vanish as soon as the main steering input initiates. Clearly, the $Q$ ratio response contained within the ellipse in figure 13 shows a consistent character resulting from the significant velocities and forces that develop continuously as a result of the steering input. At the end, it is found that the methodology is robust, provided that any investigations are carried out with reference to the steering inputs of interest.

For the test vehicle, suspension damping accounts for only a small portion of the total weight transfer which is mainly governed by an extremely stiff front anti-roll bar. As such, 
the influence of the changes made in the rear damping coefficients is not readily observable in figure 13. Figure 14 offers a magnification of the area within the ellipse and provides ample opportunity for comments. In general, the $Q$ ratio response is consistent with the character of the test-vehicle, which, including driver and co-driver exhibits a front/rear weight ratio of 53/47. A typical mild over-steering tendency is observed initially, which is attributed to the delayed response of the tyres $\left(\sigma_{y}=0.5 \mathrm{~m}\right)$. It should be noted that due to the severity of the manoeuvre the traction control was de-activated and thus no oversteering effect would be induced by traction at the rear. The assessment of damping requires further attention. To assist with the investigation, the front/rear lateral weight transfer ratio for the real and the two hypothetical set-ups is depicted in figure 15. In addition, the roll velocity is provided in figure 16. Initially, the period from 14.2 to approximately 14.4 seconds is considered. Evidently, as the rear damping increases, the front/rear lateral weight transfer ratio changes in favour of the rear end, i.e. reduces. One would expect this to result immediately in a more over-steering behaviour. However, the corresponding part in figure 14 suggests almost identical behaviour for all three vehicle setups. The prevailing explanation is that the influence of additional damping is suppressed by the tyre force lags. Nevertheless, to prove that this is the primary mechanism responsible, the combined effect of lateral weight transfer and tyre force lags should be investigated in isolation, using a much simpler model. At approximately 14.4 seconds the vehicles with increased rear damping demonstrate the expected - albeit slight - shift towards additional over-steer. However, as seen in figure 16, at approximately 14.6 seconds the roll-rate changes sign. Now, considering the induced hysteresis loop, increased damping would compensate faster for the reduction of the accumulated weight transfer at the rear. At this 
point, the front/rear weight transfer ratio increases rapidly for the vehicles with additional damping up to approximately 14.75 seconds, when their front/rear weight transfer ratio finally exceeds that of the nominal set-up. Therefore, as the vehicles reverse their roll direction, the nominal set-up is expected to exhibit a more over-steering character and this is confirmed by observing the $Q$ ratio at approximately 14.75 seconds. Apparently, by this time the tyre slip angles are well developed and all vehicles behave as dictated by the front/rear lateral weight transfer ratio. This can be justified by considering the time constant calculated for the given forward speed $(13 \mathrm{~m} / \mathrm{s})$ and the relaxation length $\left(\sigma_{y}=0.5 \mathrm{~m}\right)$. Although the tyres are not subjected to step-slip angle excitations, the time constant of approximately $0.04 \mathrm{~s}$ indicates that it is safe to assume that significant slip angles have been developed. Finally, similar comments apply to the observed change of behaviour at approximately 14.9 seconds, as a result of another change in roll direction.

\section{Concluding remarks}

The methodology presented in [6], [7], has been successfully applied for the investigation of transient handling responses, including the effects of tyre force lags and suspension damping. The use of simple 2-DOF models has highlighted the main trends with respect to tyre force lags. It is demonstrated that tyre-force-lags induce over-seer at the onset of a step-steer manoeuvre, however this behaviour soon converges to that dictated by the inherent character of the vehicle. The use of a fairly elaborate, non-linear, experimentally validated vehicle model has demonstrated the effectiveness of the approach in close-to-real situations. It is shown that the $Q$ ratio can provide with detailed information regarding the 
course of a transient manoeuvre, provided that it is used cautiously and in conjunction with other simulation outputs. The application of the methodology for the evaluation of damping effects reveals the interaction of a number of parameters. It is speculated that, initially, the influence of damping is suppressed due to the tyre force lags. As explained, this point requires further attention, possibly considering a load and/or slip dependent relaxation length [5]. In any case, the proposed approach seems capable of capturing the subtle effects of transient tyre behaviour and suspension damping which manifest their influence within the short period following a transient steering input.

\section{References}

[1] Koiter, W.T. and Pacejka, H.B. On the skidding of vehicles with one or more locked wheels. Proc. Instn. Mech. Engrs., 1968/9, 183(3H), pp 3-18.

[2] Pacejka, H. Simplified Analysis of Steady-State Turning Behaviour of Motor Vehicles.

Part 1: Handling diagrams of Simple Systems. Vehicle System Dynamics, 1973, 2, pp. 161172.

[3] Pacejka, H. Simplified Analysis of Steady-State Turning Behaviour of Motor Vehicles. Part 2: Stability of the Steady State Turn. Vehicle System Dynamics, 1973, 2, pp. 173-183.

[4] Pacejka, H. Simplified Analysis of Steady-State Turning Behaviour of Motor Vehicles. Part 3: More Elaborate Systems. Vehicle System Dynamics, 1973, 2, pp. 185-204.

[5] Pacejka, H. B. Tyre and Vehicle Dynamics, 2002 (Butterworth Heinemann), Oxford. 
[6] Mavros, G. On the objective assessment and quantification of the transient-handling response of a vehicle. Vehicle System Dynamics, 2007, 45(2), 93-112.

[7] Mavros, G., Rahnejat, H. and King, P.D. A framework for the characterisation of the transient handling responses of non-linear vehicles. Proceedings of the Institution of Mechanical Engineers, Part K: J. Multi-body Dynamics, 2007, 221(O1), 1-11.

[8] Vehicle Dynamic Terminology, SAE J670e, 1976.

[9] Ellis J.R. Vehicle Handling Dynamics, 1989, (John R. Ellis).

[10] Cook, M.V. Flight Dynamics Principles, Butterworth Heinemann, 1997

[11] Meriam, J.L. and L.G. Kraige. Engineering Mechanics Vol.1, Statics. 3 ed. Vol. 1, John Wiley, 1993

[12] Bakker, E., Pacejka, H.B. and Lidner, L. A New Tyre Model with an Application in Vehicle Dynamics Studies. SAE paper 890087, 1989.

[13] Milliken, W.F. and Milliken, D.L. Race Car Vehicle Dynamics, SAE International, 1995 


\section{APPENDIX 1}

\section{Nomenclature}

a

b

$B$

$B_{1}, B_{2}$

C

$C_{f}$

$C_{r}$

$D$

$D_{f}$

E

F

$F_{x t y r e}$

$F_{\text {ytyre }}$

$I_{w}$

$I_{x x}$

$I_{y y}$

$I_{z z}$

$I_{z x}, I_{x y}, I_{y z}$
Distance of the c.g. from the front axle

Distance of the c.g. from the rear axle

Stiffness factor (magic formula)

Boolean expressions

Shape factor (magic formula)

Cornering stiffness (front)

Cornering stiffness (rear)

Peak value (magic formula)

Front suspension damping coefficient

Curvature factor (magic formula)

Force/load

Longitudinal tyre force in the tyre SAE frame

Lateral tyre force in the tyre SAE frame

Wheel moment of inertia

Roll moment of inertia

Pitch moment of inertia

Yaw moment of inertia

Products of inertia 
$k$

$k^{\prime}$

$K_{f}$

$K_{\text {froll }}$

l

M

$m_{s}$

$m_{T}$

$p$

$q$

$Q, Q_{s}$

$R$

$r$

$S_{H}$

$S_{V}$

$T_{b}$

$T_{d}$

$t_{r f}$

U

$u$

V

$v$
Longitudinal slip ratio

Transient longitudinal slip ratio

Front wheel rate

Front anti-roll bar stiffness

Wheelbase

Moment

Sprung vehicle mass

Total vehicle mass

Roll rate

Pitch rate

Ratios

Wheel radius

Yaw rate

Horizontal shift (magic formula)

Vertical shift (magic formula)

Braking torque

Driving torque

Front track

Forward velocity

Forward speed in tyre SAE frame

Lateral velocity

Lateral speed in tyre SAE frame 


$\begin{array}{ll}W & \text { Normal velocity } \\ x_{G} & \text { Longitudinal position of c.g. in SAE frame } \\ Y & \text { Lateral tyre force function } \\ y_{G} & \text { Lateral position of c.g. in SAE frame } \\ Z & \text { Vertical displacement of vehicle body } \\ z_{G} & \text { Normal position of c.g. in SAE frame }\end{array}$

\section{Greek symbols}

$a$

$\Gamma_{d}$

$\Gamma_{k}$

$\Gamma_{k n}$

$\delta$

$\varepsilon$

$\theta$

$\rho$

$\sigma$

$\phi$

$\omega$
Slip angle

Dynamic normalised yaw impulse

Kinematic normalised yaw impulse

Neutral kinematic norm. yaw impulse

Steer angle

Neutral margin

Roll angle

Parameter (see text for definition)

Relaxation length

Pitch angle

Wheel rotational speed

\section{General subscripts}




$\begin{array}{ll}f & \text { Indicates the front of the vehicle } \\ i & \text { Indicates the } \mathrm{i}^{\text {th }} \text { corner of the vehicle } \\ r & \text { Indicates the rear of the vehicle } \\ x & \text { Longitudinal direction } \\ y & \text { Lateral direction } \\ z & \text { Normal direction }\end{array}$


Figure captions:

Figure 1: Vehicle with the SAE frame and its six motions in space

Figure 2: Experimentally obtained arbitrary-steer input excitation at approx. $13.3 \mathrm{~m} / \mathrm{s}$ forward speed

Figure 3: Comparison between measured and simulated yaw-rate

Figure 4: Comparison between measured and simulated roll-angle

Figure 5: Comparison between measured and simulated lateral acceleration

Figure 6: Comparison between measured and simulated forward speed

Figure 7: Comparison between measured and simulated vehicle path

Figure 8: The “A” term (see eq. ()) for all bicycle models

Figure 9: Q-ratio response to a 0.02 rad step-steer excitation for all bicycle models

Figure 10: Q-ratio response of the linear under-steering model for different relaxation length values

Figure 11: Components of the Q-ratio for the neutral linear model. Solid line: $\Gamma_{d}$, Dashdotted line: $\Gamma_{k n}$

Figure 12: Experimentally obtained step-steer input excitation at approx. $13 \mathrm{~m} / \mathrm{s}$ forward speed

Figure 13: Q-ratio response for the full vehicle model with three different rear damping settings

Figure 14: Magnification of area of interest from figure 13.

Figure 15: Front/rear lateral weight transfer ratio for the full vehicle model with three different rear damping settings 
Figure 16: Roll rate for the full vehicle model with three different rear damping settings

Table captions:

Table 1. Linear bicycle model parameters 


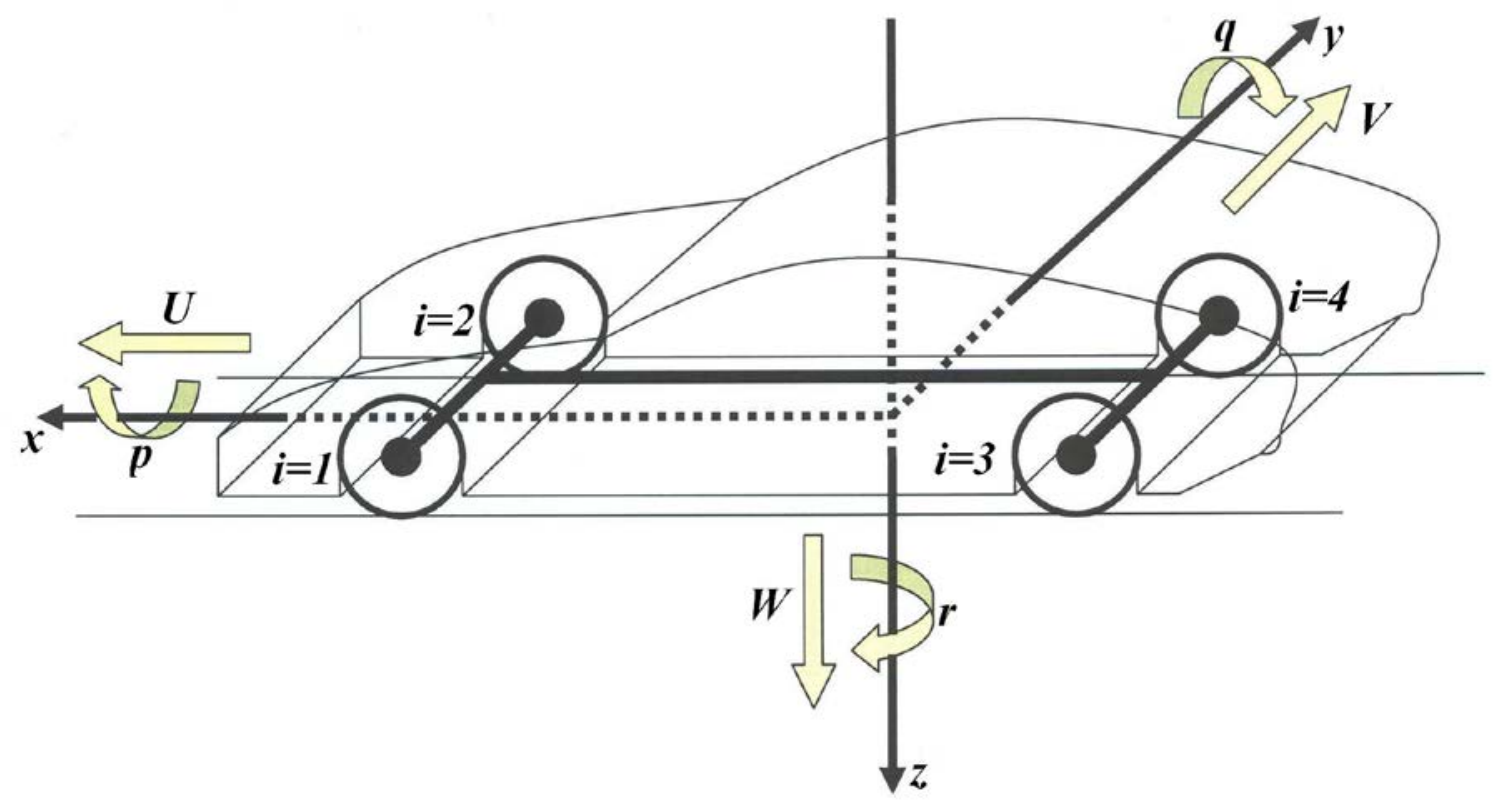

Figure 1 


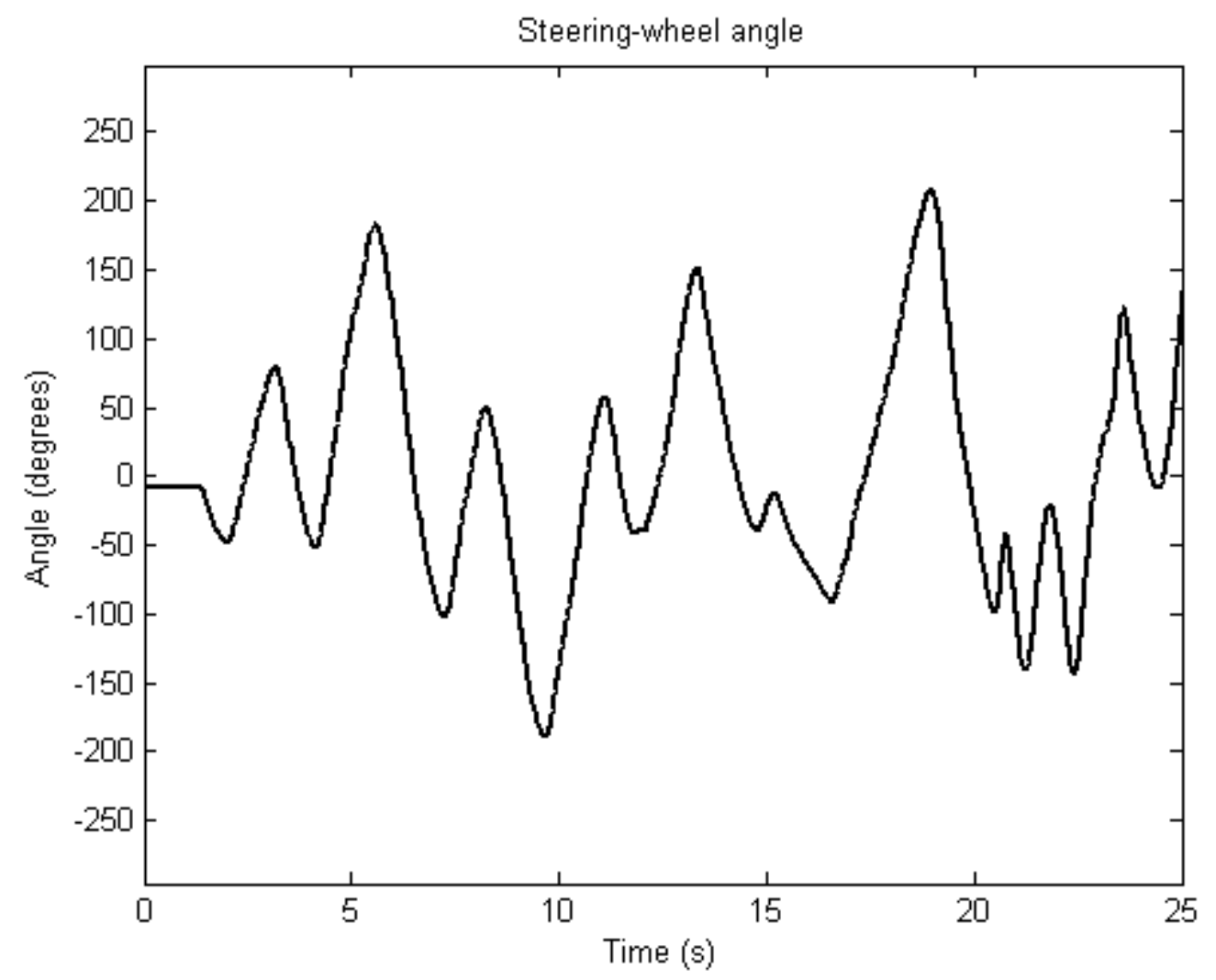

Figure 2 


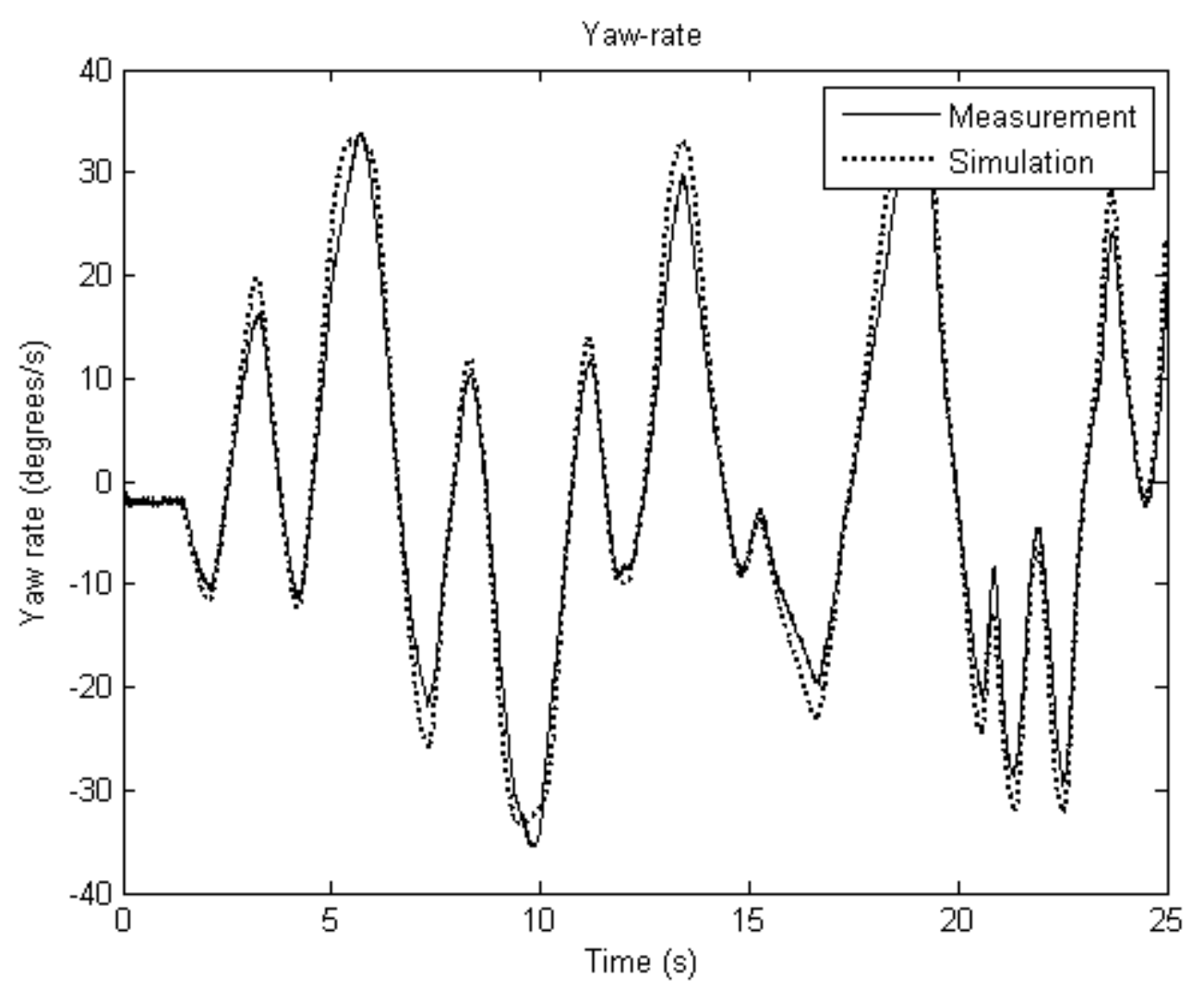

Figure 3 


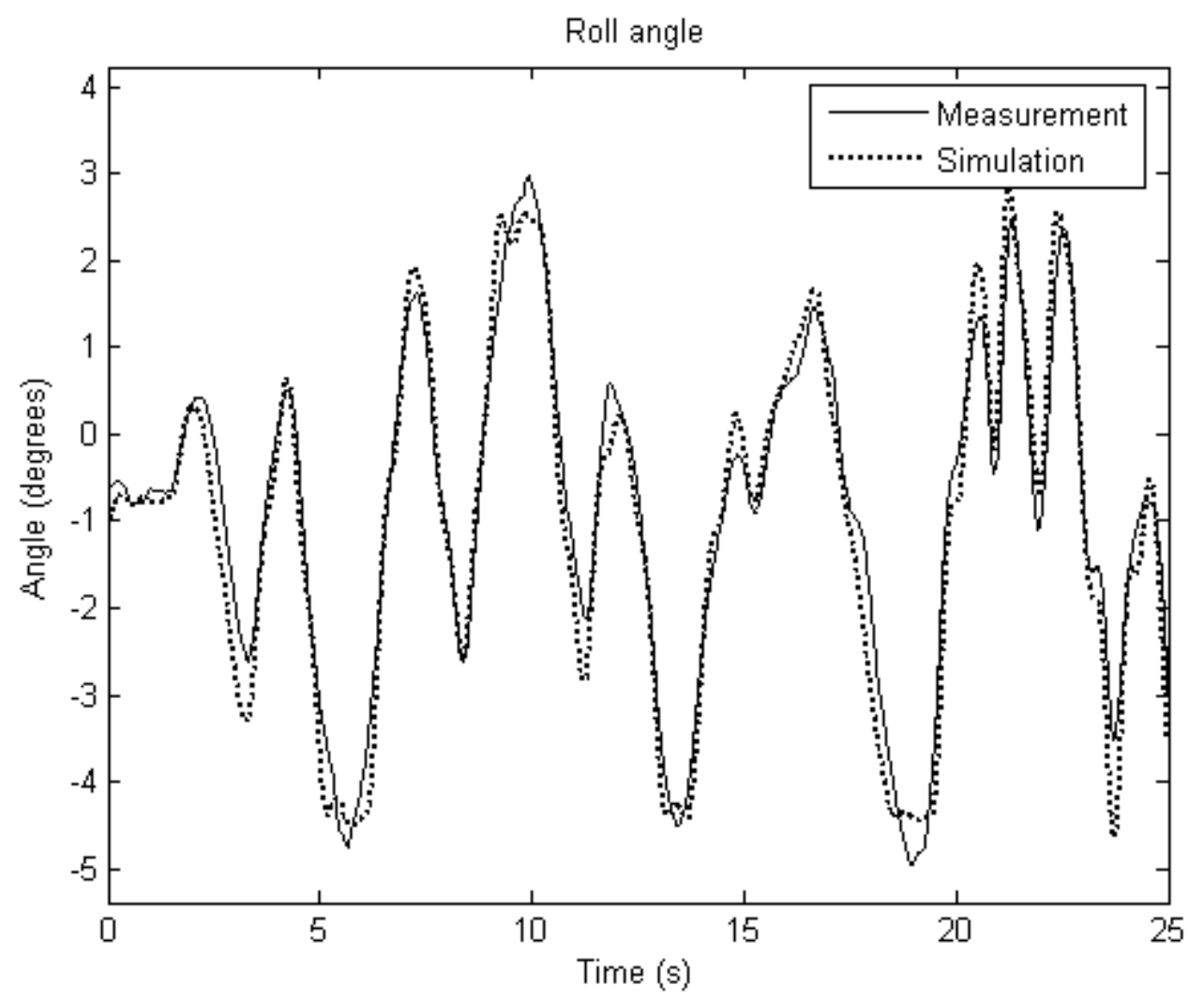

Figure 4 


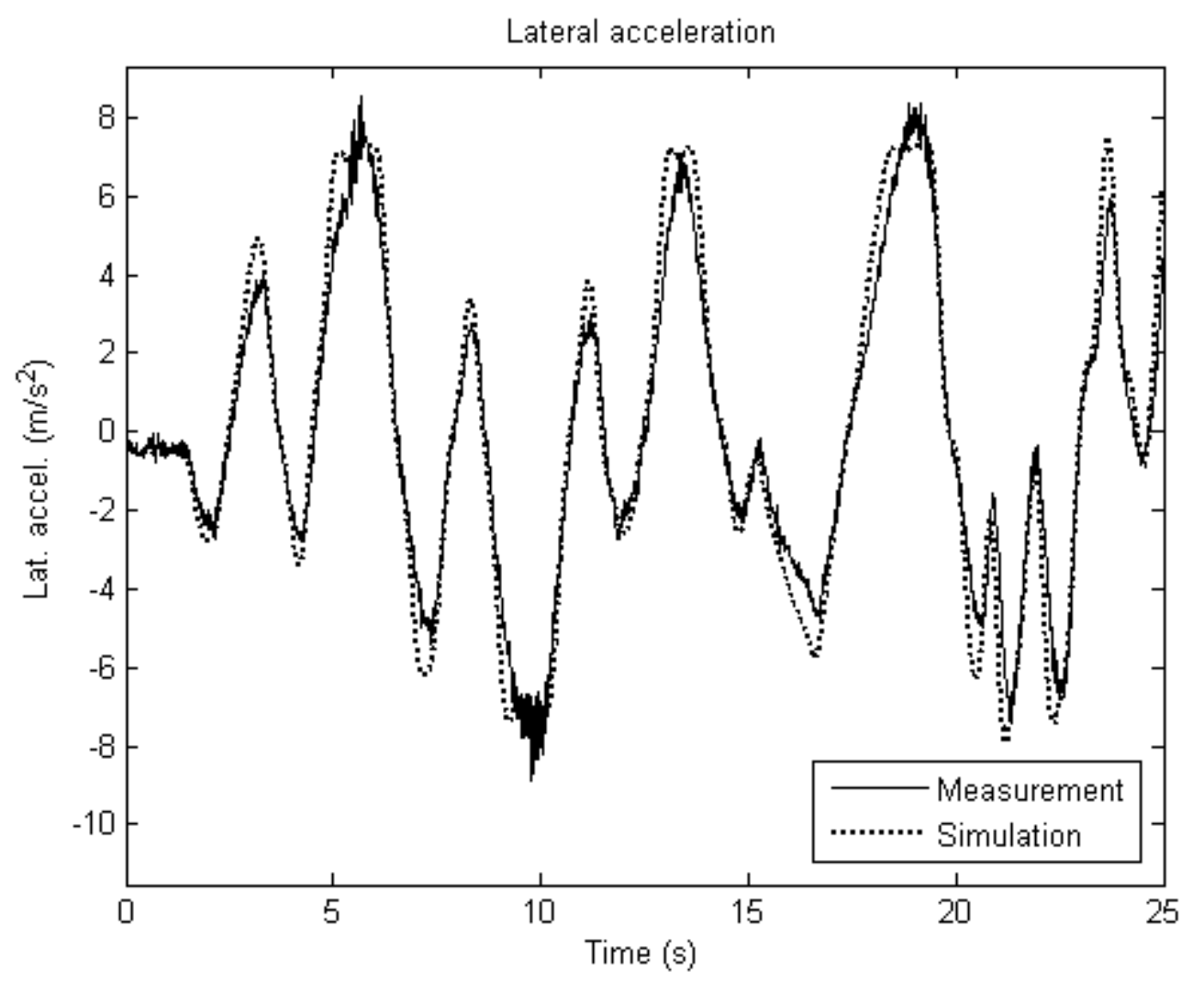

Figure 5 


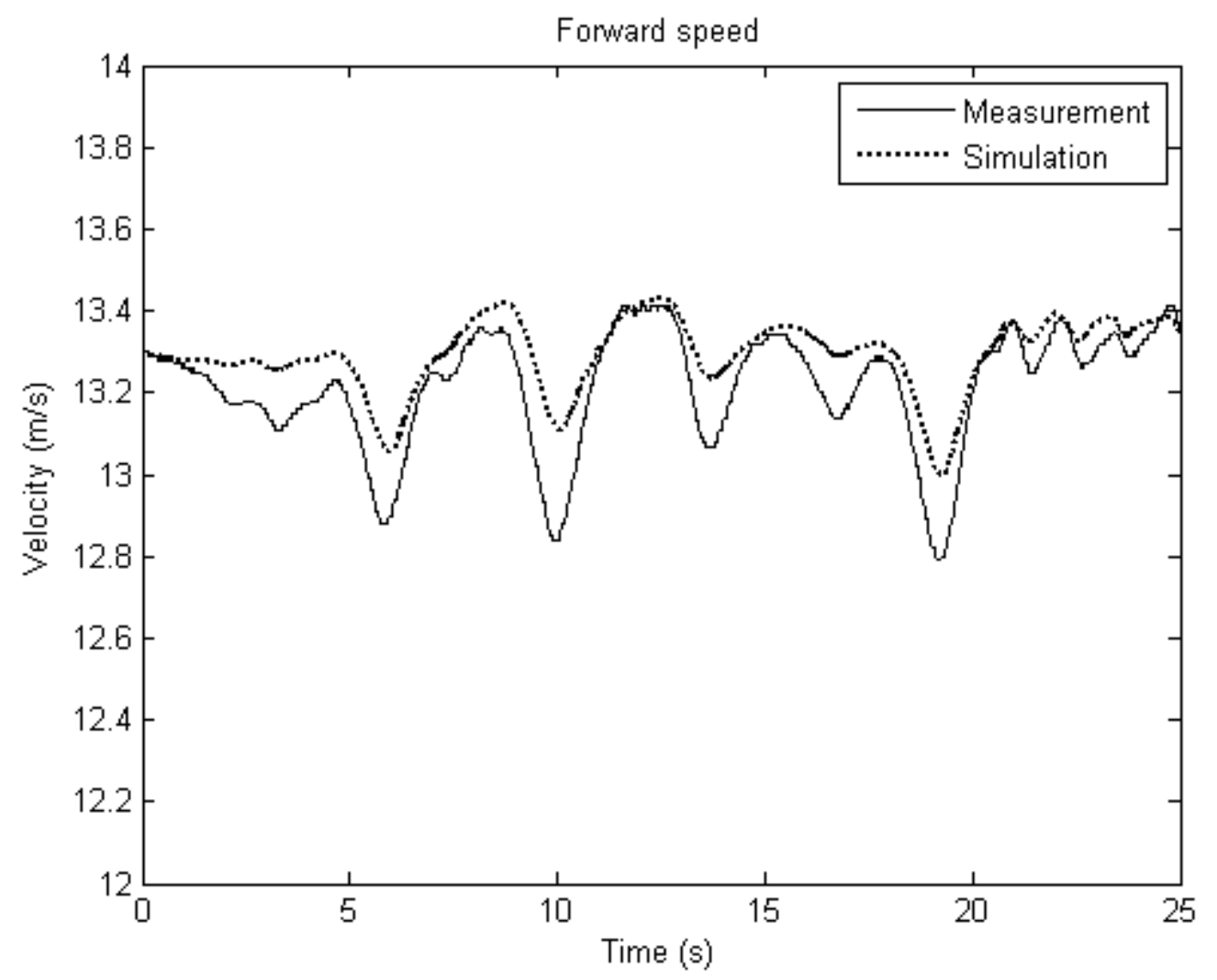

Figure 6 


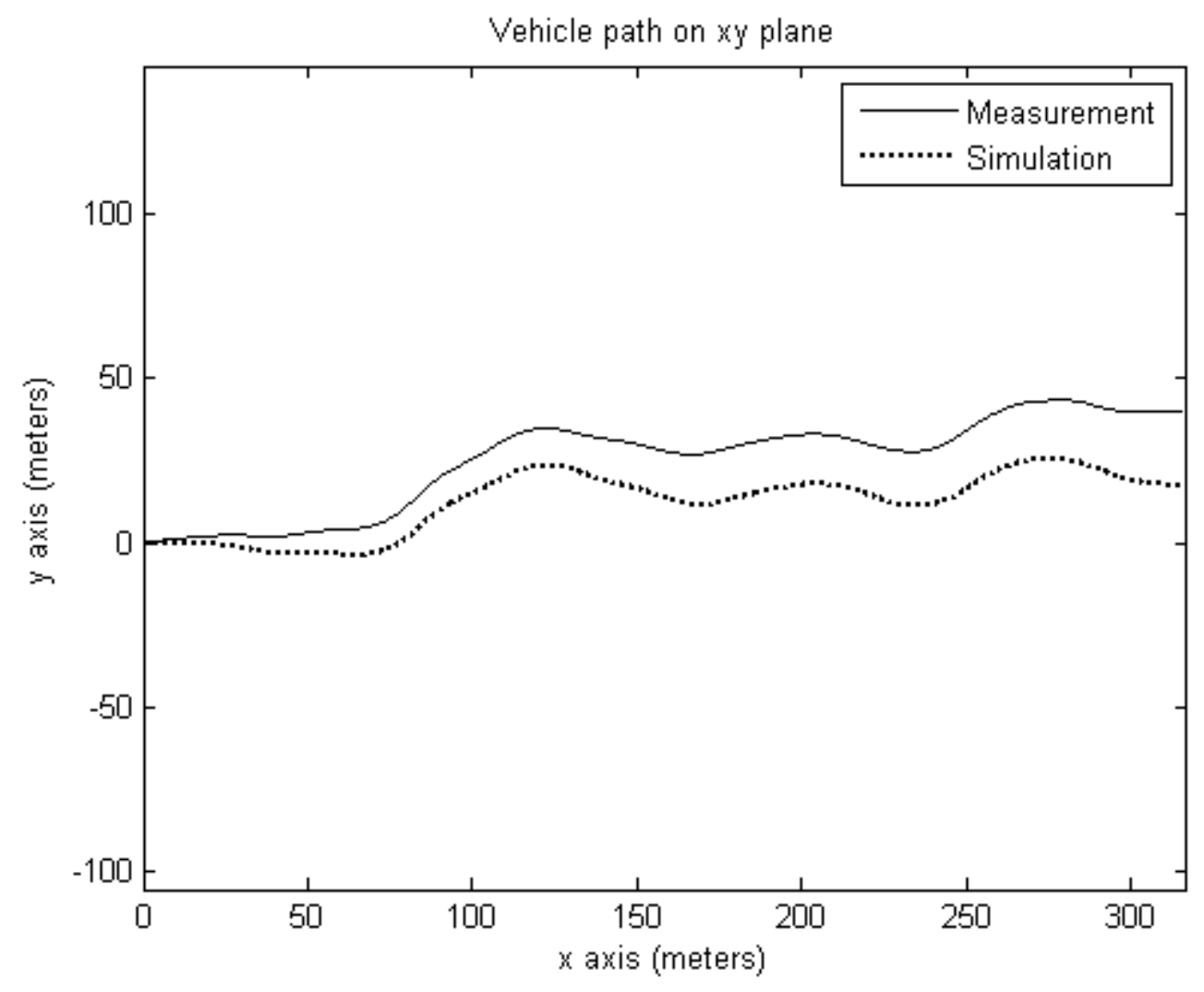

Figure 7 


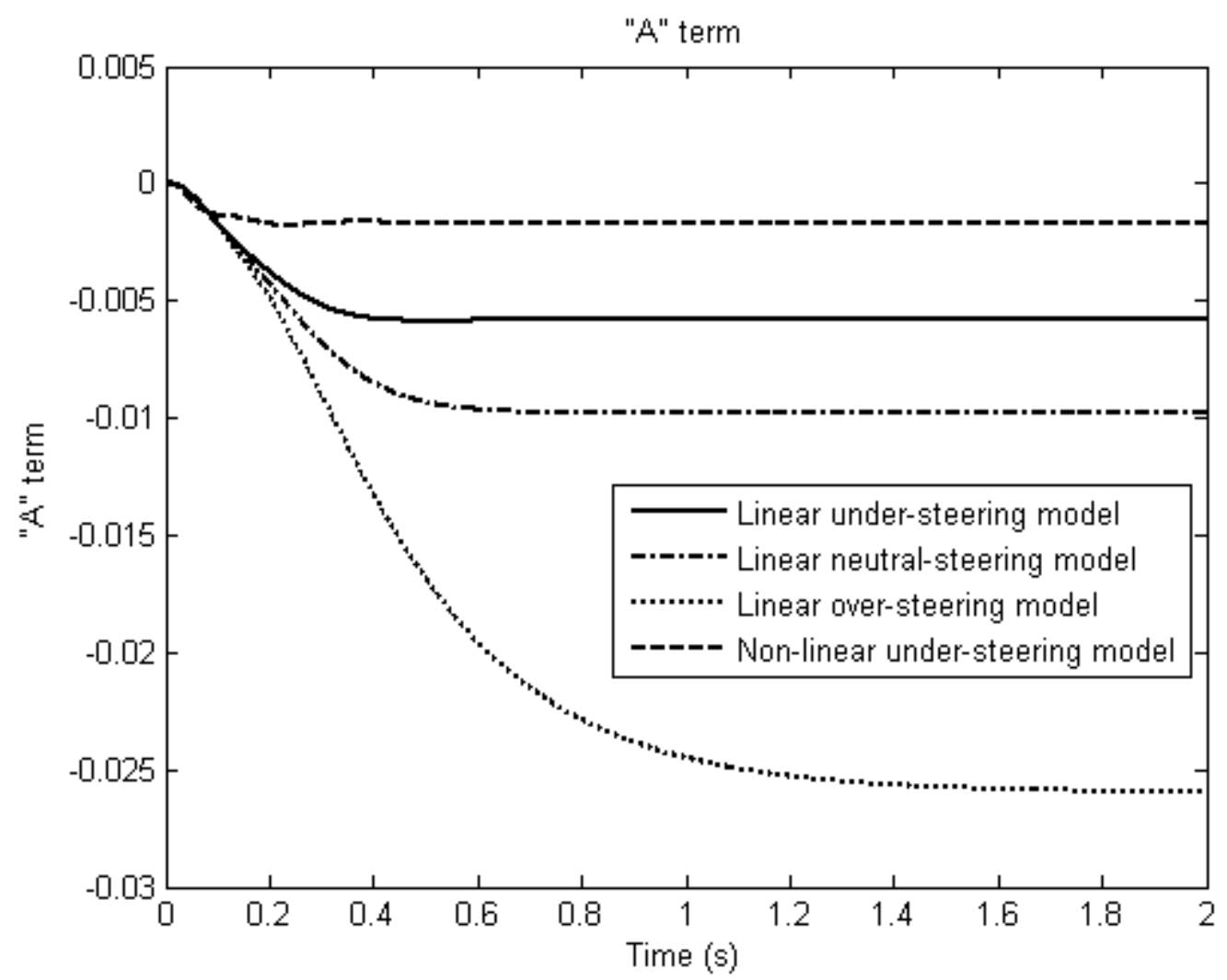

Figure 8 


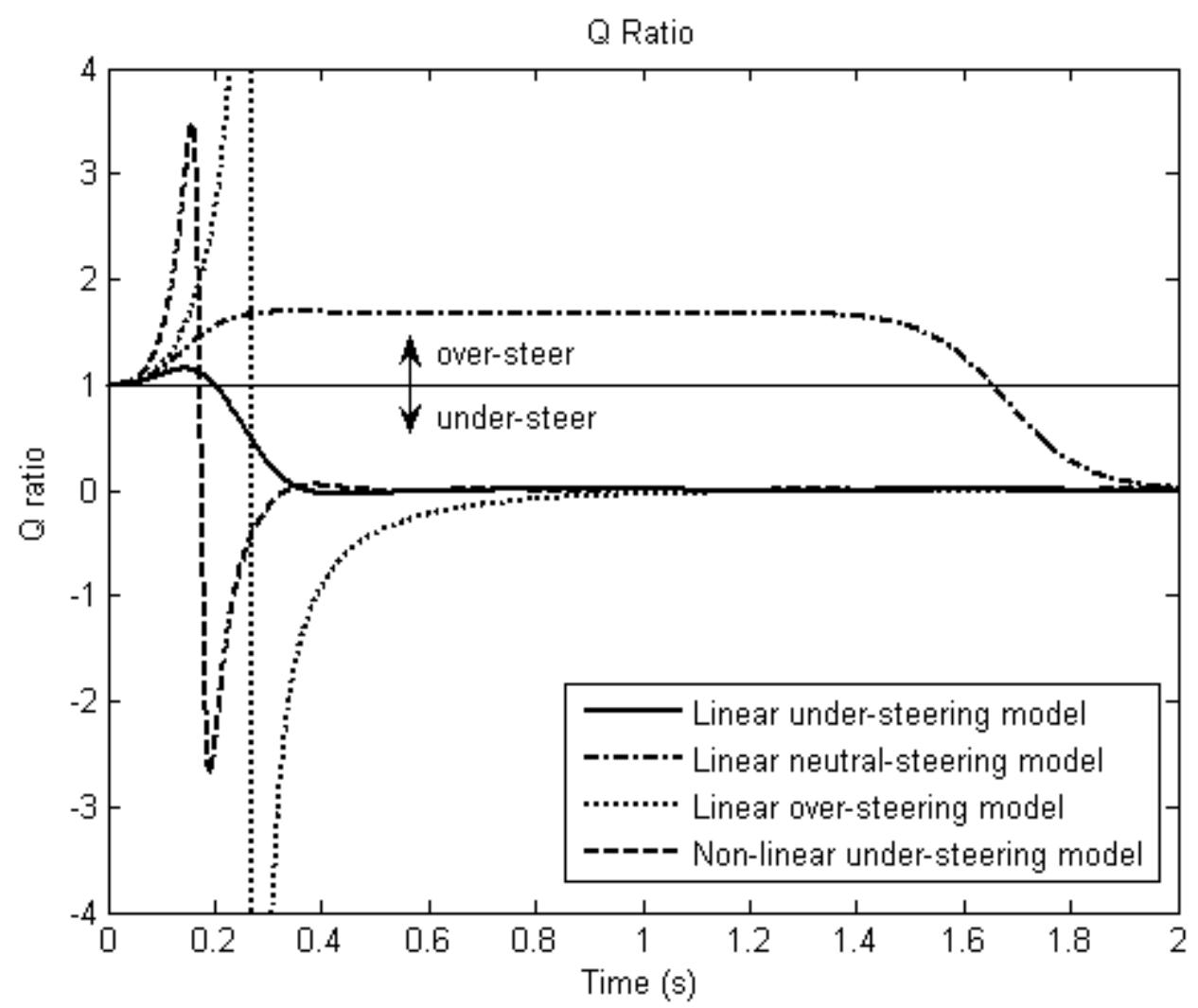

Figure 9 


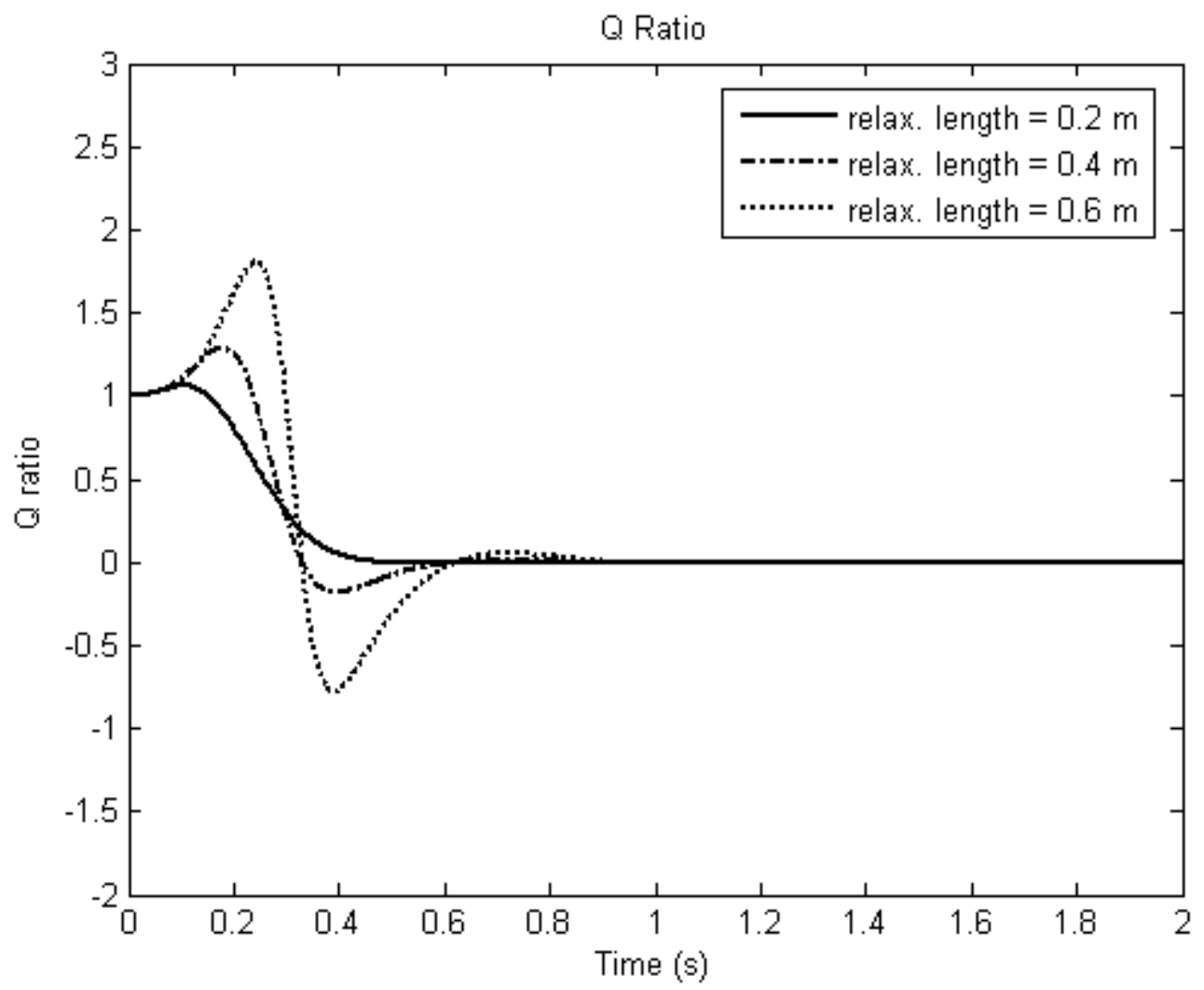

Figure 10 


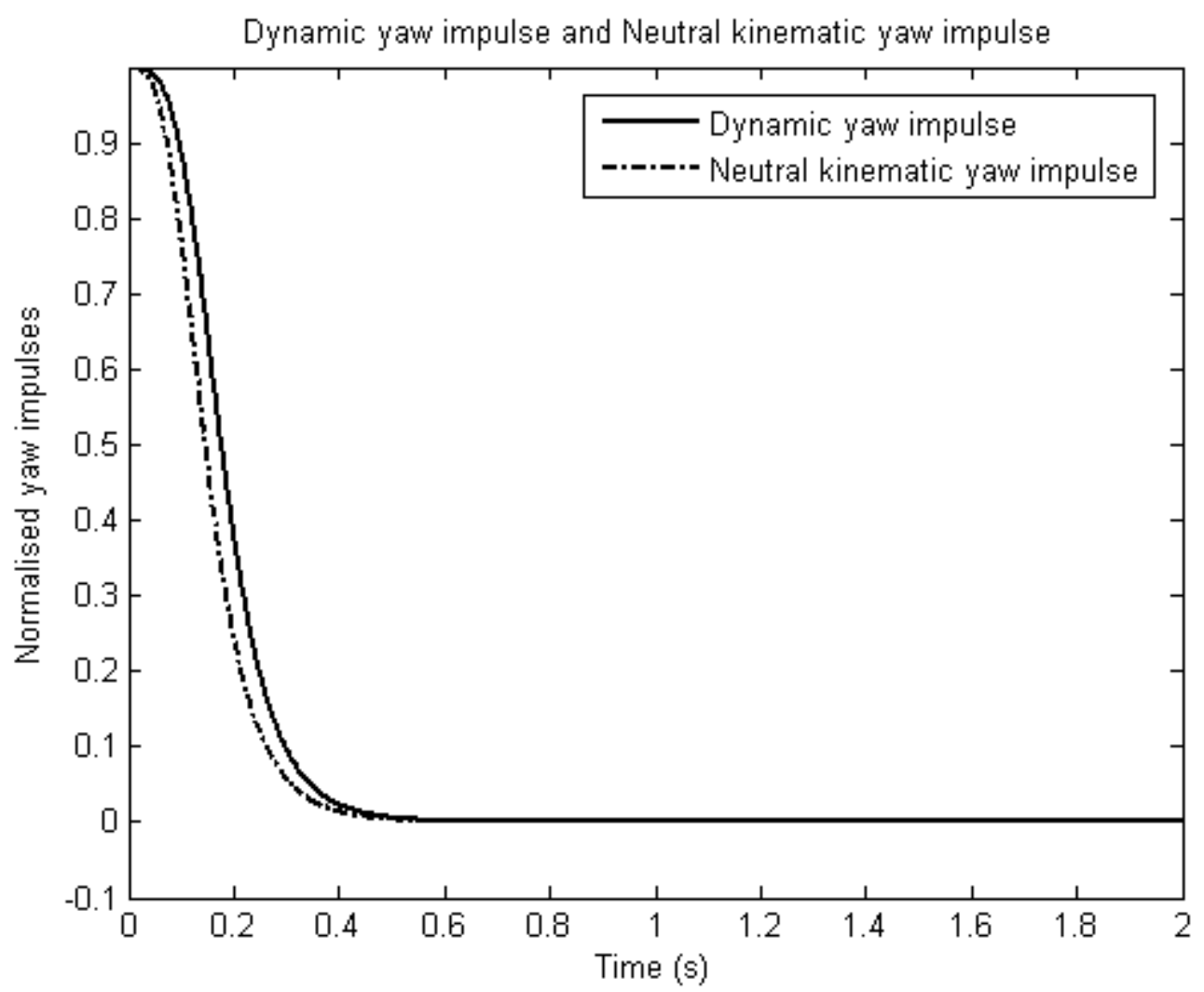

Figure 11 


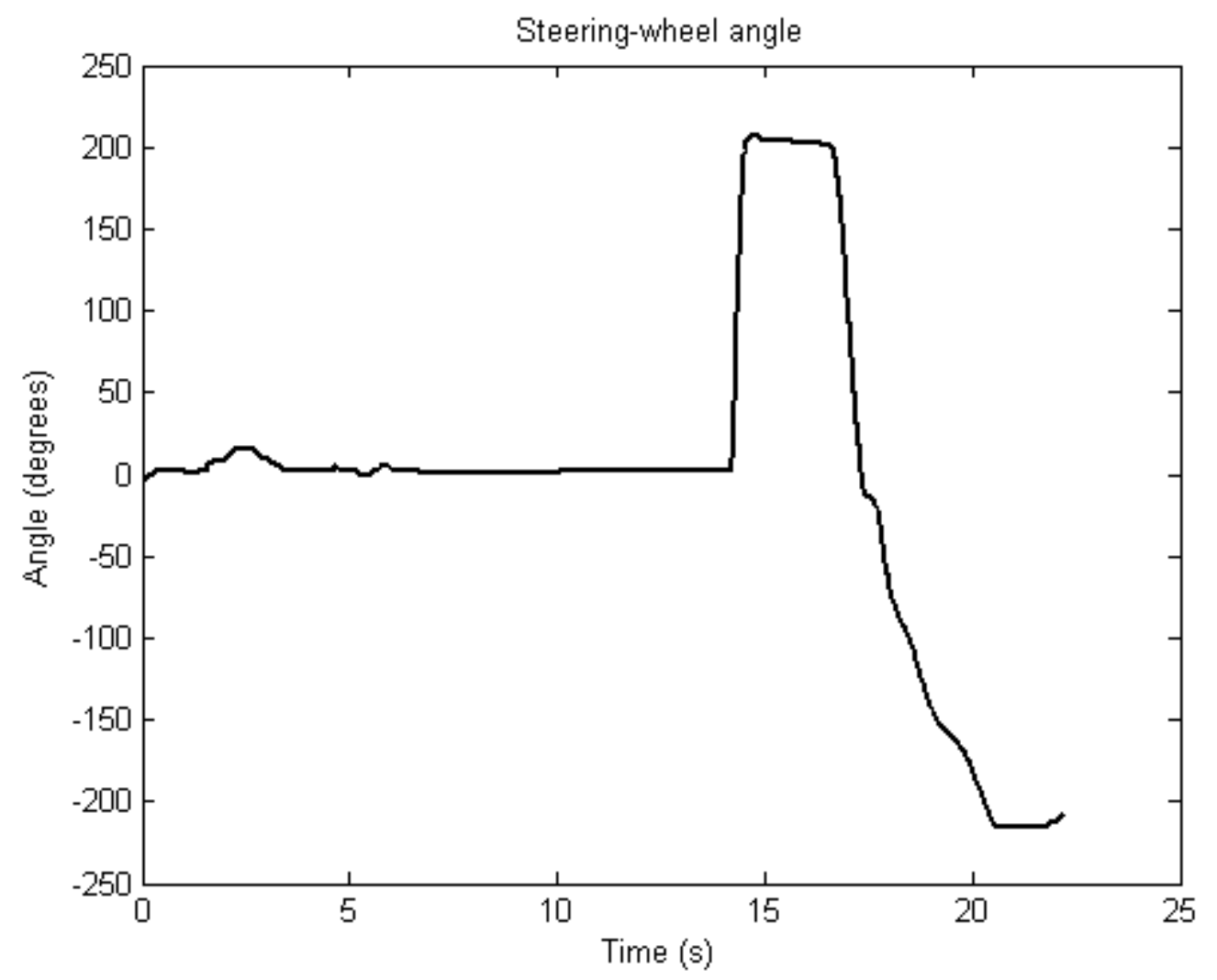

Figure 12 


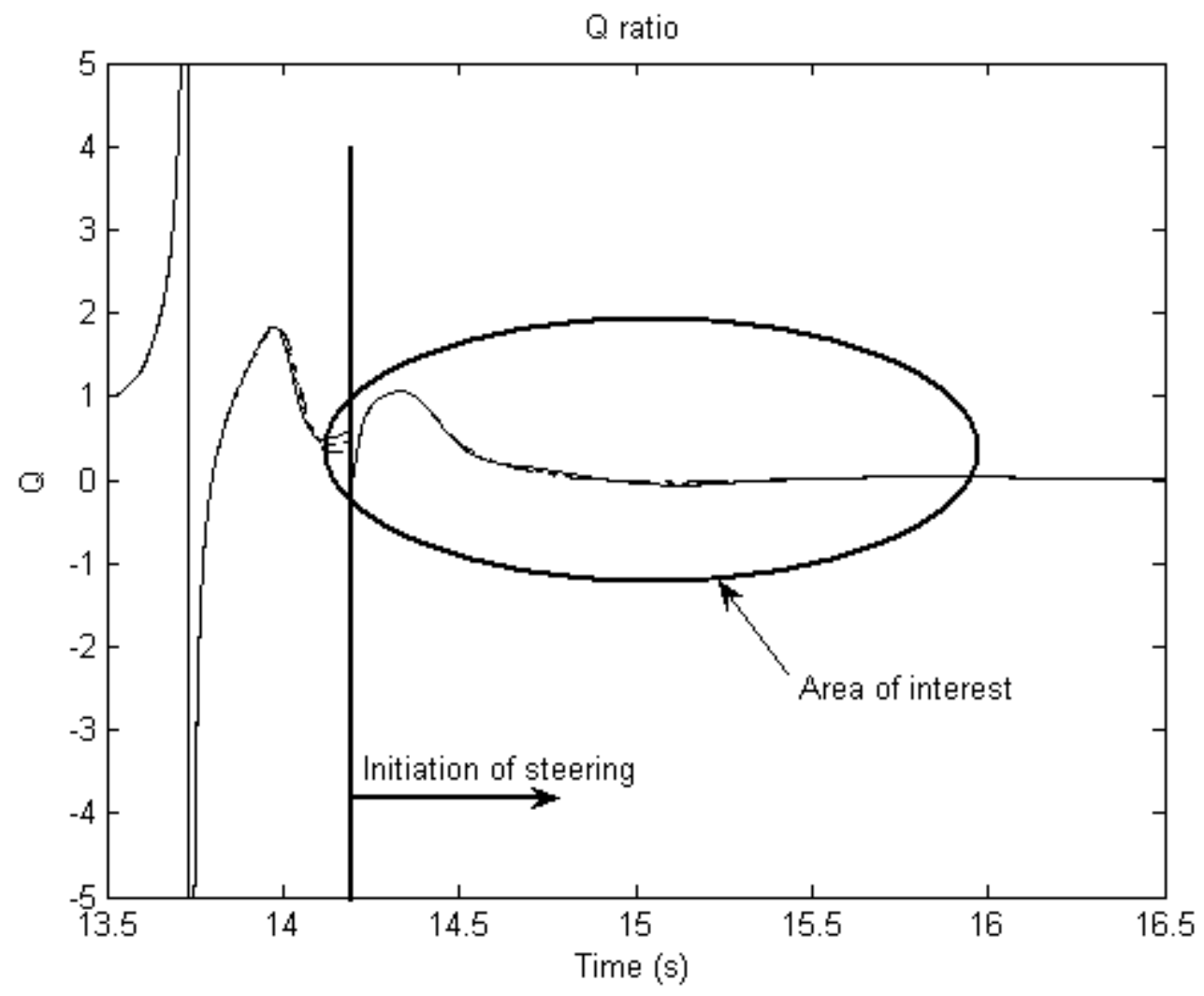

Figure 13 


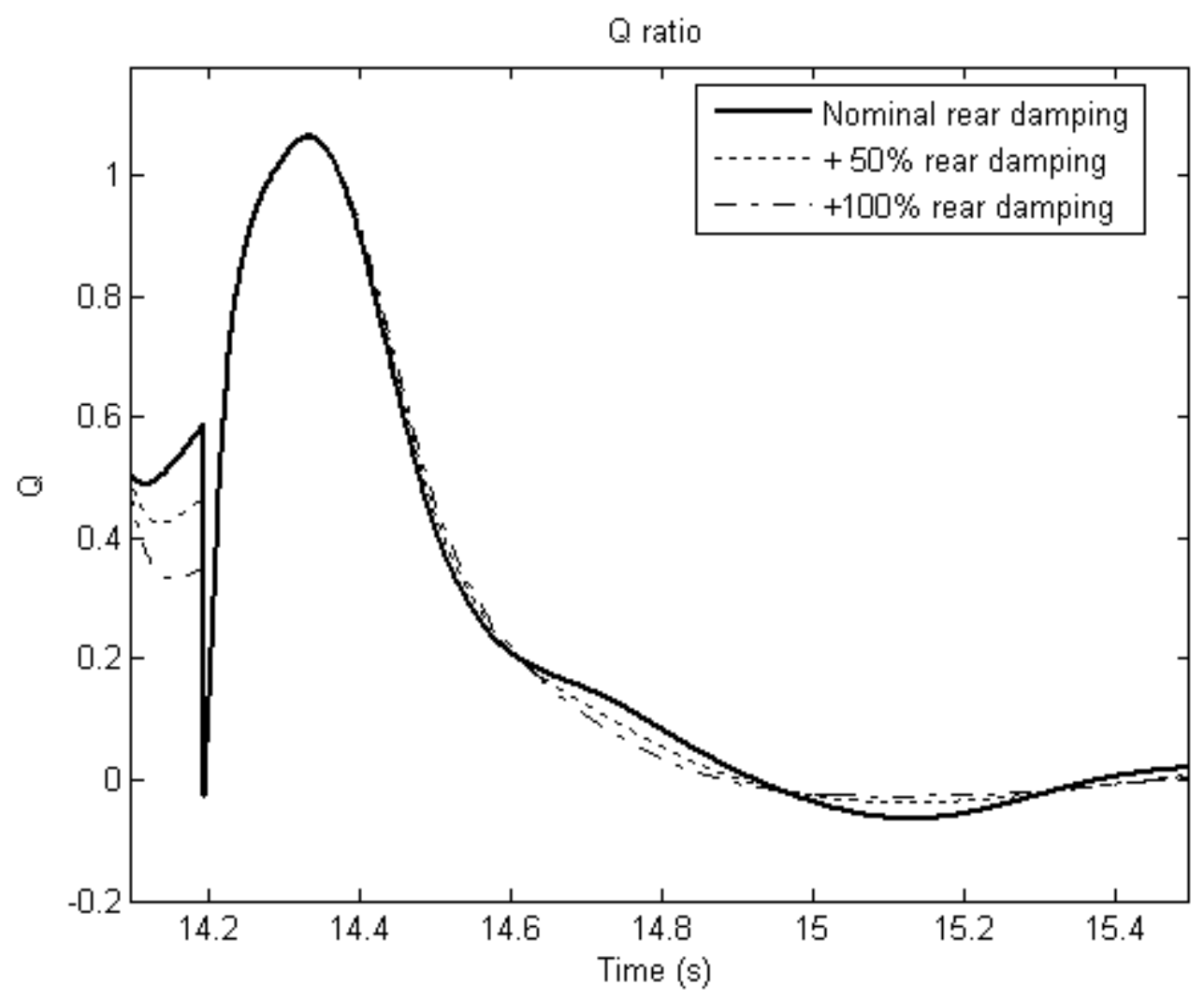

Figure 14 


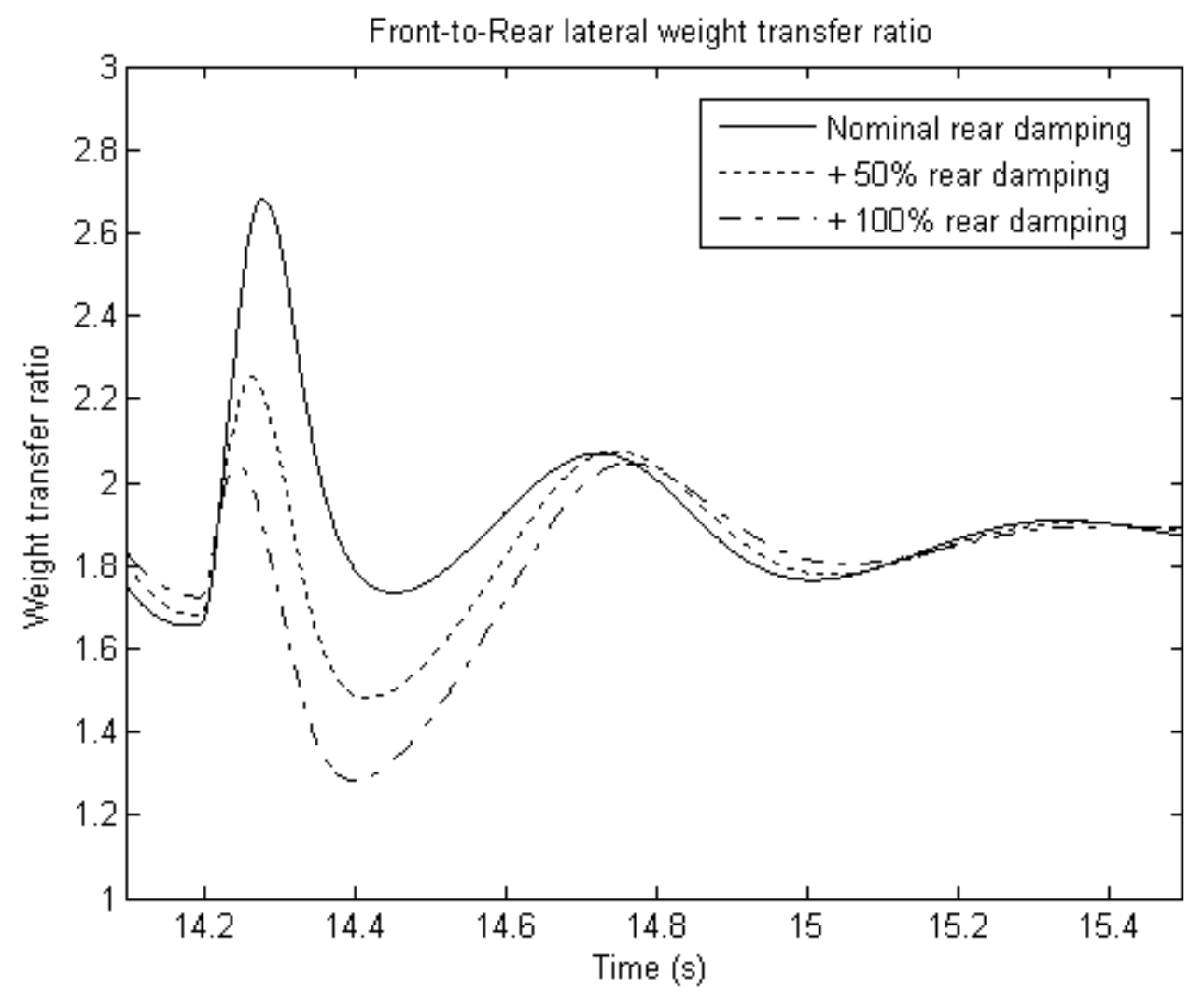

Figure 15 


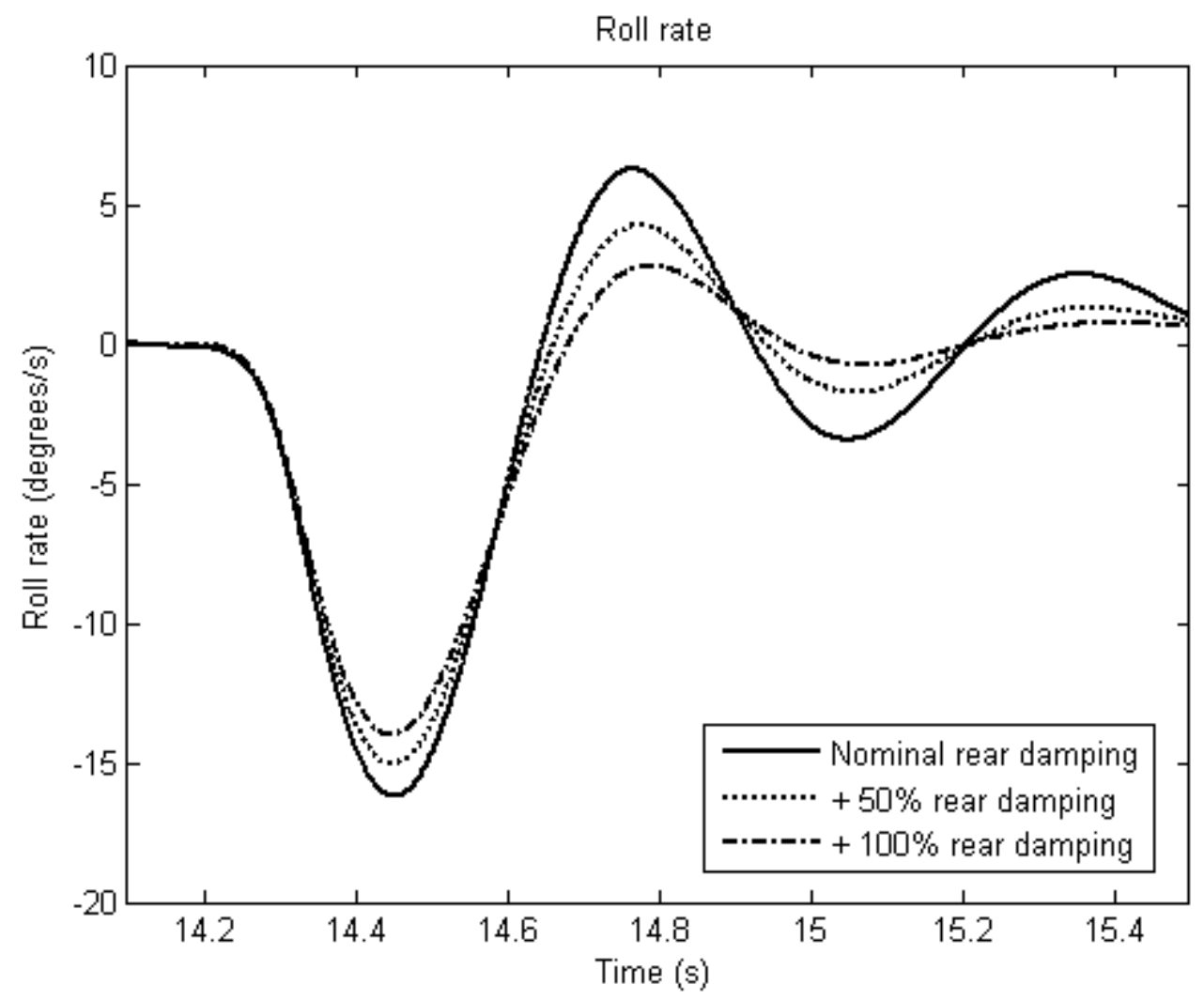

Figure 16 
Table 1

\begin{tabular}{cccccccc}
\hline $\begin{array}{c}\text { Vehicle } \\
\text { No: }\end{array}$ & $\begin{array}{c}I_{z z} \\
{\left[\mathrm{~kg} \cdot \mathrm{m}^{2}\right]}\end{array}$ & $\begin{array}{c}m \\
{[\mathrm{~kg}]}\end{array}$ & $\begin{array}{c}\mathrm{a} \\
{[\mathrm{m}]}\end{array}$ & $\begin{array}{c}\mathrm{b} \\
{[\mathrm{m}]}\end{array}$ & $\begin{array}{c}C_{f} \\
{[\mathrm{~N} / \mathrm{rad}]}\end{array}$ & $\begin{array}{c}C_{r} \\
{[\mathrm{~N} / \mathrm{rad}]}\end{array}$ & $\begin{array}{c}U \\
{[\mathrm{~m} / \mathrm{s}]}\end{array}$ \\
\hline 1 (under) & $2 \mathrm{e} 3$ & $1.3 \mathrm{e} 3$ & 1.07 & 1.43 & $6 \mathrm{e} 4$ & $6 \mathrm{e} 4$ & 10 \\
2 (neutral) & $2 \mathrm{e} 3$ & $1.3 \mathrm{e} 3$ & 1.07 & 1.43 & $6 \mathrm{e} 4$ & $4.5 \mathrm{e} 4$ & 10 \\
3 (over) & $2 \mathrm{e} 3$ & $1.3 \mathrm{e} 3$ & 1.07 & 1.43 & $6 \mathrm{e} 4$ & $3 \mathrm{e} 4$ & 10 \\
\hline \multicolumn{7}{l}{} \\
\hline
\end{tabular}

